\title{
Search for high-mass resonances in dilepton final states in proton-proton collisions at $\sqrt{s}=13 \mathrm{TeV}$
}

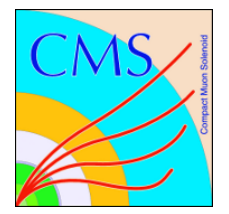

\section{The CMS collaboration}

E-mail: cms-publication-committee-chair@cern.ch

ABSTRACT: A search is presented for new high-mass resonances decaying into electron or muon pairs. The search uses proton-proton collision data at a centre-of-mass energy of $13 \mathrm{TeV}$ collected by the CMS experiment at the LHC in 2016, corresponding to an integrated luminosity of $36 \mathrm{fb}^{-1}$. Observations are in agreement with standard model expectations. Upper limits on the product of a new resonance production cross section and branching fraction to dileptons are calculated in a model-independent manner. This permits the interpretation of the limits in models predicting a narrow dielectron or dimuon resonance. A scan of different intrinsic width hypotheses is performed. Limits are set on the masses of various hypothetical particles. For the $\mathrm{Z}_{\mathrm{SSM}}^{\prime}\left(\mathrm{Z}_{\psi}^{\prime}\right)$ particle, which arises in the sequential standard model (superstring-inspired model), a lower mass limit of 4.50 (3.90) $\mathrm{TeV}$ is set at $95 \%$ confidence level. The lightest Kaluza-Klein graviton arising in the Randall-Sundrum model of extra dimensions, with coupling parameters $k / \bar{M}_{\mathrm{Pl}}$ of $0.01,0.05$, and 0.10 , is excluded at $95 \%$ confidence level below $2.10,3.65$, and $4.25 \mathrm{TeV}$, respectively. In a simplified model of dark matter production via a vector or axial vector mediator, limits at $95 \%$ confidence level are obtained on the masses of the dark matter particle and its mediator.

Keywords: Beyond Standard Model, Hadron-Hadron scattering (experiments), Lepton production, Particle and resonance production

ArXIV EPrint: 1803.06292 


\section{Contents}

1 Introduction 1

2 The CMS detector 4

3 Simulated data samples $\quad 5$

4 Lepton reconstruction and event selection $\quad 6$

$\begin{array}{llr}5 & \text { Backgrounds } & 9\end{array}$

6 Statistical analysis, results, and interpretation $\quad 11$

$\begin{array}{lll}7 & \text { Summary } & 19\end{array}$

$\begin{array}{lr}\text { The CMS collaboration } & 25\end{array}$

\section{Introduction}

Neutral resonances decaying to lepton pairs occur in a variety of theoretical models that attempt to extend the standard model (SM) of particle physics. Grand unified theories (GUTs), including superstring and left-right-symmetric models (LR), achieve unification of the three forces at a high energy scale and predict the existence of new neutral gauge bosons $[1,2]$. These bosons might be light enough to be produced at current or future colliders. Theories that allow the gravitational force to propagate into extra spatial dimensions [3] could explain the large separation between the electroweak symmetry breaking energy scale and the gravitational energy scale. In such models, graviton excitations could be observed as spin-2 high-mass resonances. Moreover, high mass neutral resonances are predicted in models where dark matter (DM), whose existence is suggested by many astrophysical and cosmological observations [4], has a particle explanation. In these theories, interactions between the DM and SM particles could be mediated by high-mass, weakly coupled particles. Indirect evidence for DM could potentially be seen at the CERN LHC by searching for a high-mass DM mediator decaying to a dilepton final state.

Typical models predicting extra Z-like bosons extend the gauge group of the SM by additional $U^{\prime}(1)$ gauge groups. The $U^{\prime}(1)$ gauge groups, or a linear combination of them, can be broken near the $\mathrm{TeV}$ scale, giving rise to new massive gauge bosons denoted as $\mathrm{Z}^{\prime}$. A generalized version of these models uses a continuously varying angle to describe the mixing of the $U^{\prime}(1)$ generators. The cross section for charged lepton pair production via a $\mathrm{Z}^{\prime}$ vector boson can, in the narrow-width approximation (NWA), be expressed in terms of the quantity $c_{\mathrm{u}} w_{\mathrm{u}}+c_{\mathrm{d}} w_{\mathrm{d}}[5,6]$. The parameter $c_{\mathrm{u}}\left(c_{\mathrm{d}}\right)$ contains information about the 


\begin{tabular}{|ccccccc|}
\hline$U^{\prime}(1)$ model & Mixing angle & $\mathcal{B}\left(\ell^{+} \ell^{-}\right)$ & $c_{\mathrm{u}}$ & $c_{\mathrm{d}}$ & $c_{\mathrm{u}} / c_{\mathrm{d}}$ & $\Gamma_{\mathrm{Z}^{\prime}} / \mathrm{M}_{\mathrm{Z}^{\prime}}$ \\
\hline $\mathrm{E}_{6}$ & & & & & & \\
$\mathrm{U}(1)_{\chi}$ & 0 & 0.061 & $6.46 \times 10^{-4}$ & $3.23 \times 10^{-3}$ & 0.20 & 0.0117 \\
$\mathrm{U}(1)_{\psi}$ & $0.5 \pi$ & 0.044 & $7.90 \times 10^{-4}$ & $7.90 \times 10^{-4}$ & 1.00 & 0.0053 \\
$\mathrm{U}(1)_{\eta}$ & $-0.29 \pi$ & 0.037 & $1.05 \times 10^{-3}$ & $6.59 \times 10^{-4}$ & 1.59 & 0.0064 \\
$\mathrm{U}(1)_{\mathrm{S}}$ & $0.129 \pi$ & 0.066 & $1.18 \times 10^{-4}$ & $3.79 \times 10^{-3}$ & 0.31 & 0.0117 \\
$\mathrm{U}(1)_{\mathrm{N}}$ & $0.42 \pi$ & 0.056 & $5.94 \times 10^{-4}$ & $1.48 \times 10^{-3}$ & 0.40 & 0.0064 \\
$\mathrm{LR}$ & & & & & & \\
$\mathrm{U}(1)_{\mathrm{R}}$ & 0 & 0.048 & $4.21 \times 10^{-3}$ & $4.21 \times 10^{-3}$ & 1.00 & 0.0247 \\
$\mathrm{U}(1)_{\mathrm{B}-\mathrm{L}}$ & $0.5 \pi$ & 0.154 & $3.02 \times 10^{-3}$ & $3.02 \times 10^{-3}$ & 1.00 & 0.0150 \\
$\mathrm{U}(1)_{\mathrm{LR}}$ & $-0.128 \pi$ & 0.025 & $1.39 \times 10^{-3}$ & $2.44 \times 10^{-3}$ & 0.57 & 0.0207 \\
$\mathrm{U}(1)_{\mathrm{Y}}$ & $0.25 \pi$ & 0.125 & $1.04 \times 10^{-2}$ & $3.07 \times 10^{-3}$ & 3.39 & 0.0235 \\
$\mathrm{GSM}$ & & & & & & \\
$\mathrm{U}(1)_{\mathrm{SM}}$ & $-0.072 \pi$ & 0.031 & $2.43 \times 10^{-3}$ & $3.13 \times 10^{-3}$ & 0.78 & 0.0297 \\
$\mathrm{U}(1)_{\mathrm{T} 3 \mathrm{~L}}$ & 0 & 0.042 & $6.02 \times 10^{-3}$ & $6.02 \times 10^{-3}$ & 1.00 & 0.0450 \\
$\mathrm{U}(1)_{\mathrm{Q}}$ & $0.5 \pi$ & 0.125 & $6.42 \times 10^{-2}$ & $1.60 \times 10^{-2}$ & 4.01 & 0.1225 \\
\hline
\end{tabular}

Table 1. Various benchmark models with their corresponding mixing angles, their branching fraction $(\mathcal{B})$ to dileptons, the $c_{\mathrm{u}}$ and $c_{\mathrm{d}}$ parameter values and their ratio, and the width to mass ratio of the associated $\mathrm{Z}^{\prime}$ boson.

model-dependent $\mathrm{Z}^{\prime}$ boson couplings to the up-type (down-type) quarks, while $w_{\mathrm{u}}\left(w_{\mathrm{d}}\right)$ depends on the up-type (down-type) quark parton distribution functions (PDFs). The parameterization of the linear mixing of the relevant $U^{\prime}(1)$ generators produces a contour in the $\left(c_{\mathrm{d}}, c_{\mathrm{u}}\right)$ plane that represents each class of models. Commonly considered models are the generalized sequential model (GSM) [6], containing the $\mathrm{Z}_{\mathrm{SSM}}^{\prime}$ boson that has SM-like couplings to SM fermions [7]; GUT models based on the $E_{6}$ gauge group, containing the $\mathrm{Z}_{\psi}^{\prime}$ boson $[1,8]$; and high-mass neutral bosons of the left (L)-right $(\mathrm{R})$ symmetric extensions of the $\mathrm{SM}$ based on the $\mathrm{SU}(2)_{\mathrm{L}} \otimes \mathrm{SU}(2)_{\mathrm{R}} \otimes \mathrm{U}(1)_{\mathrm{B}-\mathrm{L}}$ gauge group, where B-L refers to the difference between baryon and lepton numbers. Specific choices of the mixing angle produce different models such as those shown in table 1, with their exact definition given in ref. [6]. The $\left(c_{\mathrm{d}}, c_{\mathrm{u}}\right)$ plane parameterization provides a model-independent way to create a direct correspondence between the experimental bounds on $\mathrm{Z}^{\prime}$ production cross sections and the parameters of the Lagrangian. The translation of the experimental limits into the $\left(c_{\mathrm{d}}, c_{\mathrm{u}}\right)$ plane is studied both in the context of the NWA and by taking finite widths into account. The two procedures, NWA and finite widths, have been shown to give the same results [6]. A further study including the effects of interference [9] has demonstrated that the two procedures can still be used with an appropriate choice of the invariant mass window, within which the cross section is calculated.

Searches for high-mass Z' gauge bosons have been performed by the CMS Collaboration at the LHC with proton-proton collision data collected at $\sqrt{s}=7 \mathrm{TeV}[10,11]$ and with data collected at $8 \mathrm{TeV}[12,13]$. More recently CMS performed a search using the combination 
of 2015 data collected at $13 \mathrm{TeV}$ with data collected at $8 \mathrm{TeV}$ [14]. Searches for high-mass $\mathrm{Z}^{\prime}$ gauge bosons have also been performed by the ATLAS Collaboration with data collected at $7 \mathrm{TeV}[15,16]$, with data collected at $8 \mathrm{TeV}$ [17], and with data collected at $13 \mathrm{TeV}$ [18].

Kaluza-Klein graviton $\left(\mathrm{G}_{\mathrm{KK}}\right)$ excitations arising in the Randall-Sundrum (RS) model of extra spatial dimensions $[3,19]$ involve a finite five-dimensional bulk that is warped as a function of the position of the four-dimensional subspace in the fifth dimension. In particular, the RS model predicts excited Kaluza-Klein modes of the graviton, without suppressing its couplings to the SM particles. The modes appear as spin-2 resonances and can decay into dilepton final states. There are two free parameters in the model: the mass of the first graviton excitation and the coupling $k / \bar{M}_{\mathrm{Pl}}$, where $k$ is the warp factor of the five-dimensional anti-de Sitter space and $\bar{M}_{\mathrm{Pl}}$ is the reduced Planck mass. The intrinsic widths of the first excitation of the gravitons for the coupling parameters $k / \bar{M}_{\mathrm{Pl}}$ of $0.01,0.05$, and 0.10 , are $0.01,0.36$ and $1.42 \mathrm{GeV}$, respectively. Results of searches for resonances in pp collision data have previously been reported by the ATLAS and CMS Collaborations [12, 17]. At the Tevatron, the CDF and D0 Collaborations have published results based on a p $\overline{\mathrm{p}}$ collision sample at $\sqrt{s}=1.96 \mathrm{TeV}$, corresponding to an integrated luminosity of approximately $5 \mathrm{fb}^{-1}[20-25]$.

We also consider a simplified model with a single DM particle that has sizeable interactions with the SM fermions through an additional spin-1 high-mass particle mediating the SM-DM interaction. In this model, the mediator is a vector or an axial-vector boson and is exchanged in the $s$ channel $[26,27]$. There are five free parameters in this model: the DM mass $m_{\mathrm{DM}}$, the mediator mass $m_{\mathrm{Med}}$, the coupling $g_{\mathrm{DM}}$ between the mediator and the DM particle, and the universal couplings $g_{\ell}$ and $g_{\mathrm{q}}$ between the mediator and the SM charged leptons and quarks, respectively. These five parameters define the production rate of the mediator, its DM and leptonic/hadronic decay rates, and the kinematic distributions of the signal events. We investigate two sets of benchmark coupling values that illustrate the complementary strengths of dijet and dilepton searches and the typical impact of searches for dilepton resonances in this model [26]:

- vector mediator with small couplings to leptons: $g_{\mathrm{q}}=0.1, g_{\mathrm{DM}}=1.0, g_{\ell}=0.01$;

- axial-vector mediator with equal couplings to quark and leptons: $g_{\mathrm{DM}}=1.0$, $g_{\mathrm{q}}=g_{\ell}=0.1$.

Possible interference between the mediator of the dilepton process and the Drell-Yan (DY) background is well below $5 \%$ and can be safely neglected in the present analysis [26].

The results presented in this paper are obtained from an analysis of the data sample collected in 2016 at $\sqrt{s}=13 \mathrm{TeV}$, corresponding to an integrated luminosity of $35.9 \mathrm{fb}^{-1}$ for the dielectron channel and $36.3 \mathrm{fb}^{-1}$ for the dimuon channel. The invariant mass spectra of the observed dilepton final states are scrutinized for possible deviations from the SM background predictions. Background yields are estimated with simulated samples and normalized to their relative cross sections using the highest order calculations available. The sum of these backgrounds is normalized to the observed yield in the dilepton invariant mass region of $60-120 \mathrm{GeV}$. An exception to this is the background from multijet events, 


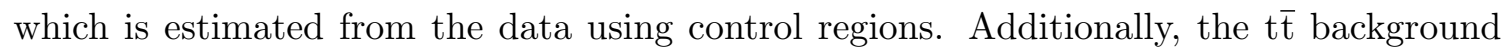
prediction from simulation is also cross-checked via e $\mu$ events in data, as discussed in section 5 .

Limits are set on the ratio of the cross section for dilepton production via a new boson to the cross section for dilepton production via the SM Z boson. This is done in order to remove the dependence on the CMS integrated luminosity measurement and to suppress the correlated uncertainties between the low- and high-mass regions. The computation of the observed limit and significance involves an arbitrary choice of the intrinsic width of the new high-mass resonance. The choice of the intrinsic width can potentially affect the statistical interpretation of the result, therefore we provide limits by scanning different width hypotheses, as discussed in section 6 . The analysis is designed to minimize the effect of the specific model assumptions on the results, allowing the results to be interpreted in the framework of any high-mass resonance decaying into lepton pairs with a width and pseudorapidity distribution similar to the reference model used. The couplings of the $\mathrm{Z}^{\prime}$ model will not only impact the width of the peak region of the high-mass resonance but also the tails due to PDFs and interference effects between the SM electroweak bosons and the new resonance in DY Z $/ \gamma^{*} \rightarrow \mathrm{e}^{+} \mathrm{e}^{-} / \mu^{+} \mu^{-}$processes. In the NWA chosen for this analysis, the contributions to the signal cross section from PDFs and interference off-shell effects, which are highly model dependent, are removed. Therefore, in this work we consider only the resonant peak, taking into account the effects of the intrinsic width of the high-mass resonance on the experimentally observed mass peak region.

This paper is structured as follows. Section 2 contains a short description of the CMS detector. In section 3, we discuss the data sample and the Monte Carlo (MC) event generators used for the signal and background simulation. The lepton reconstruction and event selection relevant for the $\mathrm{Z}^{\prime}$ boson search are discussed in section 4 . The background estimation methods are described in section 5. In section 6 the statistical method used to extract the results and the statistical treatment of the systematic uncertainties are explained. Results are summarized in section 7 .

\section{The CMS detector}

The central feature of the CMS detector is a superconducting solenoid providing an axial magnetic field of $3.8 \mathrm{~T}$ and enclosing an inner tracker, an electromagnetic calorimeter (ECAL), and a hadron calorimeter (HCAL). The inner tracker is composed of a silicon pixel detector and a silicon strip tracker, and measures charged particle trajectories in the pseudorapidity range $|\eta|<2.5$. The ECAL and HCAL, each composed of a barrel and two endcap sections, extend over the range $|\eta|<3.0$. The finely segmented ECAL consists of nearly 76000 lead tungstate crystals, while the HCAL is constructed from alternating layers of brass and scintillator. Forward hadron calorimeters encompass $3.0<|\eta|<5.0$. The muon detection system covers $|\eta|<2.4$ with up to four layers of gas-ionization detectors installed outside the solenoid and sandwiched between the layers of the steel flux-return yoke. Additional detectors and upgrades of electronics were installed before the beginning of the $13 \mathrm{TeV}$ data collection period in 2015, yielding improved reconstruction performance 
for muons relative to the $8 \mathrm{TeV}$ data collection period in 2012. The efficiency to reconstruct and select muons that result from $\mathrm{Z}$ boson decays and pass specific identification selection criteria, has increased by approximately $2 \%$ between Run 1 and Run 2 as a result of these upgrades [28]. A more detailed description of the CMS detector, together with a definition of the coordinate system used and the relevant kinematic variables, can be found in ref. [29].

The CMS experiment has a two-level trigger system. The level-1 (L1) trigger [30], composed of custom hardware processors, selects events of interest using information from the calorimeters and muon detectors and reduces the readout rate from the $40 \mathrm{MHz}$ bunch crossing frequency to a maximum of $100 \mathrm{kHz}$. The software based high-level trigger (HLT) [30] uses the full event information, including that from the inner tracker, to reduce the event rate to around the $1 \mathrm{kHz}$ that is retained for further processing.

\section{Simulated data samples}

The dominant background in this search is the DY process. The simulated DY background is generated with POWHEG v2 [31-36] from next-to-leading order (NLO) matrix elements using the NNPDF3.0 [37] PDF set, and with PYTHIA 8.205 [38] for parton showering and hadronization. For all simulated SM samples, the default tune for PYTHIA, CUETP8M1 [39], is used. The DY cross section at NLO is corrected to next-to-next-toleading order (NNLO) in perturbative quantum chromodynamics (QCD) by using a dilepton invariant mass dependent $K$-factor according to the predictions of the FEWZ 3.1.b2 program [40]. In addition, these predictions incorporate missing EW corrections at NLO. For the FEWZ calculations, the LUXqed_plus_PDF4LHC15_nnlo_100 [41] PDF set is used in which the QCD PDFs based on the PDF4LHC [42] set are combined with the photon PDFs to account for pure quantum electrodynamics effects. Another nonresonant background arises from a $\gamma \gamma$ initial state via $t$ and $u$ channel processes. The photon-induced (PI) process produces two leptons in the final state [43, 44]. This contribution is included in the $K$-factor that corrects the DY NLO cross section.

The $t \bar{t}$, tW and WW backgrounds are simulated using POWHEG v2, with parton showering and hadronization described by PYTHIA 8.205. The NNPDF3.0 PDF set is used for

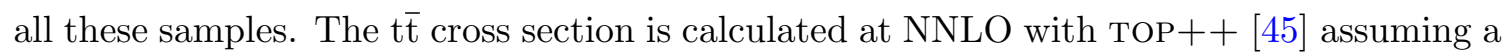
top quark mass of $172.5 \mathrm{GeV}$. The inclusive diboson processes $\mathrm{WZ}$, and ZZ are simulated at leading order (LO) using the PYTHIA 8.205 program along with the NNPDF3.0 PDFs. The production of DY $\tau^{+} \tau^{-}$and $\mathrm{W}+$ jets is simulated at LO with the MADGRAPH5_aMC@NLO version 2.2.2 [46] program. The PDFs are evaluated using the LHAPDF library [47-49].

We use a sample of events in which a $Z_{\psi}^{\prime}$ boson is generated with a mass of $3000 \mathrm{GeV}$, and RS samples with the graviton generated at different mass values from 250 to $4000 \mathrm{GeV}$. In all samples the high-mass resonances decay to electron and muon pairs. Both signal samples are generated using the PYTHIA 8.205 program with the NNPDF3.0 PDFs. The $\mathrm{Z}_{\psi}^{\prime}$ sample is used to create simulated peaks in the dilepton mass plots of figure 1. However, we use DY samples to model the $\mathrm{Z}^{\prime}$ at high masses, since the dilepton behaviour in this region is identical in the two cases. 
The presence of additional pp interactions in the same or adjacent bunch crossing observed in data (pileup) is incorporated in simulated events by including overlapping pp interactions. MC samples are corrected to reproduce the pileup distribution as measured in data (pileup reweighting), with an average number of pileup interactions per proton bunch crossing of approximately 22 for the 2016 data sample. The detector response is simulated using the GEANT4 [50] package.

\section{Lepton reconstruction and event selection}

The electron and muon reconstruction algorithms and event selection criteria used in this high-mass dilepton search are mostly unchanged from the previous analysis [14]. However, the muon selection criteria were modified in both the online and offline selection in order to increase the efficiency in the high mass region, above $1 \mathrm{TeV}$.

Energy deposits in the ECAL are combined into clusters under the assumption that each local maximum represents a single particle. Any clusters consistent with originating from a single particle that may have undergone bremsstrahlung emission are grouped together. If a track from the nominal interaction point is geometrically associated with a cluster, this track together with the cluster form an electron candidate. The angular information of the electron candidate is taken from the track. The energy of the electron uses only the ECAL deposits and is not combined with the track momentum. Electron candidates are required to have a transverse momentum $p_{\mathrm{T}}>35 \mathrm{GeV}$ and satisfy $\left|\eta_{C}\right|<1.44$ (ECAL barrel region) or $1.57<\left|\eta_{C}\right|<2.50$ (ECAL endcap region), where $\eta_{C}$ is the pseudorapidity of the cluster of ECAL deposits comprising the electron with respect to the nominal centre of the CMS detector. The transition region $1.44<\left|\eta_{C}\right|<1.57$ is excluded as it leads to lower-quality reconstructed clusters, owing mainly to services and cables exiting between the barrel and endcap calorimeters.

The electron candidates are also required to pass a set of dedicated high energy electron selection criteria [51]. This selection requires that the lateral spread of deposits in the ECAL be consistent with that of a single electron, that the track be matched to the ECAL deposits and be consistent with a particle originating from the nominal interaction point, and that the associated energy in the HCAL around the electron direction be less than $5 \%$ of the reconstructed energy of the electron, once noise and pileup are taken into account. The selection also requires that the electron be isolated in a cone of radius $\Delta R=\sqrt{(\Delta \eta)^{2}+(\Delta \phi)^{2}}=0.3$ in both the calorimeter and tracker [14]. Only well-measured tracks that are consistent with originating from the same vertex as the electron are included in the isolation sum.

The efficiency of the trigger to select events with two electrons passing the analysis selection requirements is $98.5 \%$, when the barrel (endcap) electrons satisfy $p_{\mathrm{T}}>36(38) \mathrm{GeV}$. This primary trigger is monitored by a suite of higher-threshold triggers which have progressively fewer selection requirements, culminating in a trigger that simply requires $800 \mathrm{GeV}$ of $p_{\mathrm{T}}$ in the ECAL. These triggers are included to minimize the chance that unexpected reconstruction problems cause a lower than expected efficiency in the primary trigger.

In the selection of dielectron pairs, at least one of the two electrons must be in the ECAL barrel region in order to reduce the background from multijet events. This also 
allows the endcap-endcap events to be used as a control sample for the QCD background estimate. Dielectron pairs are not required to be oppositely charged as this leads to a significant efficiency loss at high invariant mass [10]. As the electron energy is solely obtained from the calorimeter, an incorrectly measured charge does not impact the measured mass. If there are multiple possible dielectron pairs, the pair with the two highest $p_{\mathrm{T}}$ electrons is selected.

The efficiency to trigger, reconstruct, and select an electron pair with invariant mass equal to $1 \mathrm{TeV}$ within the detector acceptance is 69 (65)\% for barrel-barrel (barrel-endcap) events. The trigger efficiency is measured in data and the total efficiency is estimated using simulated DY events and validated using data measurements at the $\mathrm{Z}$ boson mass peak. Using $\mathrm{Z}$ bosons, it is possible to probe the efficiencies up to electron $p_{\mathrm{T}}$ of $500 \mathrm{GeV}$ within a few percent precision. The uncertainty in the efficiency is $\pm 3( \pm 5) \%$ in the barrel (endcap) for electrons coming from a dielectron pair with a mass $m_{\ell \ell}>120 \mathrm{GeV}$. The simulated efficiency reproduces the energy evolution of the observed efficiency in the measurable region from 40 to $500 \mathrm{GeV}$.

A candidate muon pair at the L1 trigger is required to have at least one muon reconstructed with segments in the muon detectors and transverse momentum $p_{\mathrm{T}}$ above $22 \mathrm{GeV}$. These muons are then required to have $p_{\mathrm{T}}>50 \mathrm{GeV}$ and $|\eta|<2.4$ at the HLT. A prescaled HLT path is used to extract the observed yield in the invariant mass region of the $\mathrm{Z}$ boson peak $\left(60<m_{\ell \ell}<120 \mathrm{GeV}\right)$ in order to construct the normalization factor to that region. This prescaled trigger has a $p_{\mathrm{T}}$ threshold of $27 \mathrm{GeV}$ with the same $\mathrm{L} 1$ requirements as the main trigger.

The trigger efficiency for dimuon events, where both muons have $p_{\mathrm{T}}>53 \mathrm{GeV}$, is parameterized using simulated DY events. It is measured to be around $99.5 \%$ if both muons are in the barrel $(|\eta|<1.2)$, and $99.0 \%$ for events with one muon in the endcap $(|\eta|>1.2)$. These efficiencies are validated as a function of muon $p_{\mathrm{T}}$ and $\eta$, using data events that pass the full offline selection criteria. This is done using muons present in high mass dilepton or high- $p_{\mathrm{T}} \mathrm{Z}$ boson events (free from background contributions) and other data sets (selected by electron, missing transverse momentum, or jet requirements). The measurements are found to be in agreement for muons with $p_{\mathrm{T}}$ up to $1.5 \mathrm{TeV}$ and an uncertainty of \pm 0.3 ( \pm 0.7$) \%$ for barrel-barrel (barrel-endcap and endcap-endcap) dimuon events over the full mass range is assigned.

High- $p_{\mathrm{T}}(>200 \mathrm{GeV})$ muon offline reconstruction uses dedicated algorithms [52] to take into account the effects of radiative processes of high-energy muon interactions with the detector material. Muon candidates are required to have $p_{\mathrm{T}}>53 \mathrm{GeV}$, to be within the region of $|\eta|<2.4$, and to pass dedicated high-momentum identification selection criteria [13]. Muon candidates are also required to pass isolation requirements. The summed $p_{\mathrm{T}}$ of tracks within a cone of radius $\Delta R=0.3$ around the candidate direction should be less than $10 \%$ of the $p_{\mathrm{T}}$ of the candidate, excluding from summation the muon candidate under consideration.

Oppositely charged muon candidates passing the selection are combined to form dimuon candidates. A $\chi^{2}$ fit is performed to the common vertex between the two muons to ensure that they originate from the same vertex. This fit is required to have reduced 
$\chi^{2}<20$. The angle between the directions of the two muon candidates is required to be less than $\pi-0.02$ in order to suppress cosmic ray backgrounds. If there are multiple possible dimuon pairs, the pair with the two highest $p_{\mathrm{T}}$ muons is selected.

Standalone muon reconstruction and identification efficiencies as a function of the muon momentum, both in data and simulation, were probed using high quality isolated inner tracks extrapolated to the muon system. In the barrel region $\mathrm{MC}$ efficiencies are validated up to $1.2 \mathrm{TeV}$, while in the endcaps we observed a small discrepancy with respect to data at the order of $10 \%$ for muon momentum of $3 \mathrm{TeV}$. The efficiency ratios between data and MC obtained as a function of the single muon momentum are then propagated as a function of the dimuon mass. It leads to $-1.5(-6.5) \%$ one-sided uncertainties on a $4 \mathrm{TeV}$ mass for barrel-barrel (barrel-endcap and endcap-endcap) events. This represents the dominant uncertainty in the signal acceptance times efficiency.

The efficiency to trigger, reconstruct, and select a muon pair with invariant mass equal to $1 \mathrm{TeV}$ within the detector acceptance is $92.7_{-0.5}^{+0.3 \%}\left(92.5_{-2.7}^{+0.7} \%\right)$ for barrel-barrel (barrelendcap and endcap-endcap) events. The efficiency for each of the two muons is correlated as is the case for electron pairs.

The experimental dilepton mass resolution is determined from simulation as a function of the generated dilepton mass. The simulated mass resolution measured in simulations is smeared in order to be comparable to $\mathrm{Z}$ boson events selected in data. This smearing is performed for electrons in the entire $p_{\mathrm{T}}$ range while for the muon channel this smearing is performed as a function of the leading muon $p_{\mathrm{T}}$ up to $800(450) \mathrm{GeV}$ in the barrelbarrel (barrel-endcap and endcap-endcap) events. The experimental mass resolution is 1.0 (1.5)\% for barrel-barrel (barrel-endcap) electron pairs with a mass of $1 \mathrm{TeV}$. No uncertainty is assigned to the electron pair mass resolution as we use the resolution measured in the low-mass region, which also represents the most pessimistic value for higher masses. The resolution for muon pairs with a mass of $1 \mathrm{TeV}$ is $3.0 \pm 0.5(4.0 \pm 0.6) \%$ for barrel-barrel (barrel-endcap and endcap-endcap) events. We assign a $15 \%$ systematic uncertainty in these values, based on measurements with different functions to parameterize the resolution for muon pairs.

The response of the detector to leptons might depend on the increasing dilepton invariant mass. For electrons this could reflect a nonlinear response of the readout electronics. There is no evidence for such effects in the current data. The energy scale uncertainty at high $p_{\mathrm{T}}(400 \mathrm{GeV})$ is validated at the $2(1) \%$ level for electrons in the barrel (endcaps). Since the calorimeter is expected to behave linearly with energy we therefore assume this is true for a $2 \mathrm{TeV}$ mass. As the muon $p_{\mathrm{T}}$ increases, it becomes increasingly sensitive to the detector alignment. New methods have been developed for the 2016 data to determine a potential bias from this source. The usage of muon alignment position uncertainties has been added in the HLT and offline muon reconstruction algorithms in order to compensate for misalignment. The curvature distributions of positive and negative muons $\left(q / p_{\mathrm{T}}\right)$ in data are compared to those obtained in simulation for different $\eta$ and $\phi$ ranges. The mass scale is within 1 (3)\% for barrel-barrel (barrel-endcap and endcap-endcap) dimuon events for the entire mass range up to $2.3 \mathrm{TeV}$, covering the region where dimuon events are observed. 


\section{$5 \quad$ Backgrounds}

The dominant SM background arises from the DY process. In addition to the DY process, $\mathrm{e}^{+} \mathrm{e}^{-}$and $\mu^{+} \mu^{-}$pairs can be produced in the PI process $\gamma \gamma \rightarrow \ell^{+} \ell^{-}$[43] from photons radiated by the incoming protons. The PI process was studied through investigations of proton PDFs that include photon contributions [43, 44]. Although the relative contribution of PI processes increases with dilepton mass, the effect on the statistical analysis of the data was found to be negligible. Uncertainties in the PDFs, in the contributions from PI processes, and in the NNLO corrections to the cross sections dominate the systematic uncertainty in the amount of estimated background. This in turn dictates the uncertainty in the background shape. The uncertainty due to the PDFs is assessed using the PDF4LHC prescription [42] and is found to vary from 1.4 to $20 \%$ as the dilepton mass increases from 0.2 to $6 \mathrm{TeV}$.

Other sources of background are real leptons from the top quark-antiquark $(\mathrm{t} \overline{\mathrm{t}})$, single top quark (tW), diboson (WW, WZ, and ZZ), and DY $\tau^{+} \tau^{-}$processes. The contribution of these sources is reduced at high invariant dilepton mass. These backgrounds are estimated

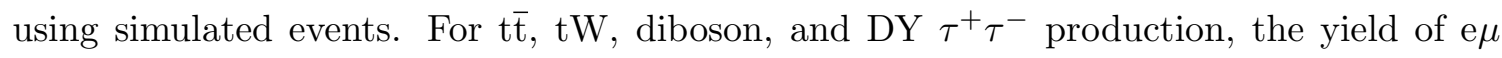
final states produced should be approximately equal to the sum of ee and $\mu \mu$ final states. Therefore the simulation predictions in these channels are compared to data in the $\mathrm{e} \mu$ final state. The predictions from data and simulation are in agreement within uncertainties and no further action is taken.

Multijet-enriched data control samples are used to evaluate the contribution of jets misidentified as electrons, as described in ref. [13]. Similarly, multijet data control samples are used to evaluate the probability for jets and nonisolated muons to be identified as isolated muons. The contribution of misidentified jets to the total background is $1-3 \%$; therefore even with large uncertainties up to $50 \%$, it has a negligible effect on the statistical analysis of the data.

The contribution of cosmic ray background events is negligible in this analysis due to the event selection, and is $<0.2 \%$ for muons with $p_{\mathrm{T}}>300 \mathrm{GeV}$.

The observed invariant mass spectra of the dielectron and dimuon events are presented in figure 1. The highest observed dilepton mass is $2.6 \mathrm{TeV}$ and appears in the dielectron final state as a barrel-endcap event. The highest observed dimuon mass is $2.3 \mathrm{TeV}$ and appears as a barrel-endcap event. The structure observed just above the $\mathrm{Z}$ boson peak in the dimuon channel is due to the high threshold $\left(p_{\mathrm{T}}>53 \mathrm{GeV}\right)$ applied to the transverse momentum of the muon candidates. The uncertainty bands in the ratio plots represent the systematic uncertainty in the background yields arising from the dilepton mass scale, trigger efficiency, acceptance times efficiency, Z boson normalization ( $1 \%$ for dielectron and $5 \%$ for dimuon channel), PDFs, non-DY background cross section determination (7\%), and lepton misidentification rates, summed in quadrature. The selection criteria for the dielectron channel have a small sensitivity to pileup, so the contribution from pileup reweighting is included in the uncertainty band. The dimuon selection criteria are by construction insensitive to pileup.

The corresponding cumulative distributions are shown in figure 2. The SM expected yields in various mass bins are compared to the observed yields in tables 2 and 3. Ob- 

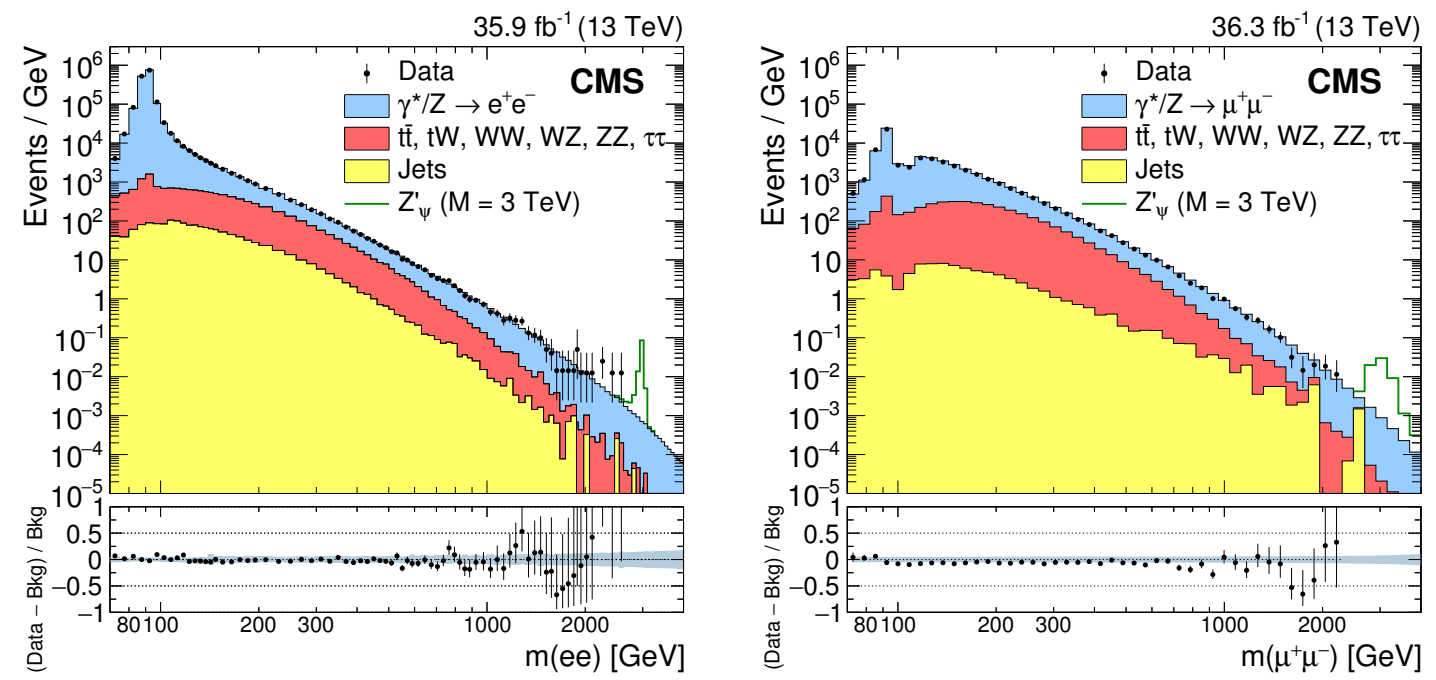

Figure 1. The invariant mass spectra of dielectron (left) and dimuon (right) events. The points with error bars represent the observed yield. The histograms represent the expectations from the $\mathrm{SM}$ processes. The bins have equal width in logarithmic scale so that the width in $\mathrm{GeV}$ becomes larger with increasing mass. Example signal shapes for a narrow resonance with a mass of $3 \mathrm{TeV}$ are shown by the stacked open histograms. The uncertainty bands in the ratio plots represent the systematic uncertainty in the background yields.

\begin{tabular}{|c|c|c|c|c|c|}
\hline $\begin{array}{c}m_{\mathrm{ee}} \text { range } \\
{[\mathrm{GeV}]}\end{array}$ & $\begin{array}{c}\text { Observed } \\
\text { yield }\end{array}$ & $\begin{array}{c}\text { Total } \\
\text { background }\end{array}$ & $\mathrm{Z} / \gamma^{*}$ & $\begin{array}{c}\mathrm{t} \overline{\mathrm{t}}+\text { other } \\
\text { backgrounds }\end{array}$ & $\begin{array}{l}\text { Jet mis- } \\
\text { reconstruction }\end{array}$ \\
\hline $120-400$ & 245101 & $252000 \pm 13000$ & $199000 \pm 11000$ & $47700 \pm 2100$ & $5800 \pm 2900$ \\
\hline $400-600$ & 4297 & $4430 \pm 230$ & $2890 \pm 150$ & $1400 \pm 88$ & $137 \pm 69$ \\
\hline $600-900$ & 943 & $986 \pm 64$ & $739 \pm 49$ & $221 \pm 17$ & $26 \pm 13$ \\
\hline $900-1300$ & 182 & $187 \pm 14$ & $156 \pm 12$ & $26.8 \pm 2.3$ & $3.9 \pm 1.9$ \\
\hline 1300-1800 & 33 & $34.3 \pm 3.4$ & $30.9 \pm 3.2$ & $2.8 \pm 0.5$ & $0.6 \pm 0.3$ \\
\hline$>1800$ & 9 & $7.5 \pm 1.1$ & $7.0 \pm 1.1$ & $0.30 \pm 0.04$ & $0.13 \pm 0.07$ \\
\hline
\end{tabular}

Table 2. The number of dielectron events in various invariant mass ranges. The total background is the sum of the events for the SM processes listed. The yields from simulation are normalized relative to the expected cross sections, and overall the simulation is normalized to the observed yield using the number of events in the mass window $60-120 \mathrm{GeV}$. Uncertainties include both statistical and systematic components, summed in quadrature.

servations agree with expectations in the entire mass region for the dielectron events. A deficit of dimuon events is observed in the high-mass region compared to the expectations from SM processes (as shown on the right plot of figure 2). This deficit appears in the barrel $(|\eta|<1.2)$. In the barrel-barrel category we observe two dimuon candidates in the region $m_{\mu \mu}>1600 \mathrm{GeV}$, where we expect ten dimuon candidates from MC simulations. This leads to a local significance of the discrepancy equal to 2.9 standard deviations (s.d.). This significance is reduced to 1.8 s.d. when considering the entire pseudorapidity range and is considered to be compatible with a statistical fluctuation. No experimental sources for the deficit were identified. 

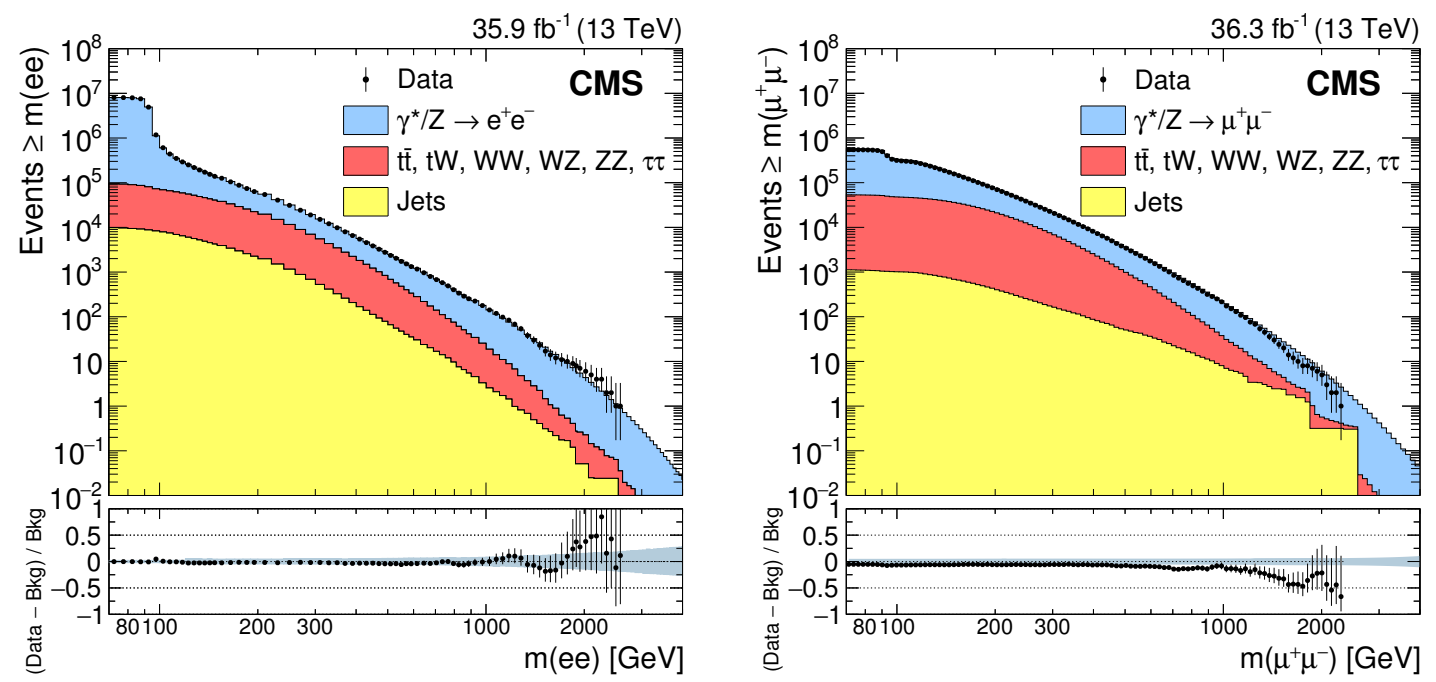

Figure 2. The cumulative distributions, where all events above the specified mass on the $x$ axis are summed, of the invariant mass spectra of dielectron (left) and dimuon (right) events. The points with error bars represent the observed yield. The histograms represent the expectations from SM processes. The uncertainty bands in the ratio plots represent the systematic uncertainty in the background yields.

\begin{tabular}{|c|c|c|c|c|c|}
\hline $\begin{array}{c}m_{\mu^{+} \mu^{-}} \text {range } \\
{[\mathrm{GeV}]}\end{array}$ & $\begin{array}{c}\text { Observed } \\
\text { yield }\end{array}$ & $\begin{array}{c}\text { Total } \\
\text { background }\end{array}$ & $\mathrm{Z} / \gamma^{*}$ & $\begin{array}{c}\mathrm{t} \overline{\mathrm{t}}+\text { other } \\
\text { backgrounds }\end{array}$ & $\begin{array}{c}\text { Jet mis- } \\
\text { reconstruction }\end{array}$ \\
\hline $120-400$ & 244277 & $260000 \pm 14000$ & $218000 \pm 11000$ & $40900 \pm 3500$ & $800 \pm 400$ \\
\hline $400-600$ & 5912 & $6290 \pm 350$ & $4340 \pm 230$ & $1900 \pm 160$ & $50 \pm 25$ \\
\hline $600-900$ & 1311 & $1430 \pm 80$ & $1070 \pm 60$ & $340 \pm 30$ & $20 \pm 10$ \\
\hline $900-1300$ & 244 & $268 \pm 15$ & $220 \pm 12$ & $41 \pm 4$ & $7 \pm 4$ \\
\hline $1300-1800$ & 41 & $50 \pm 3$ & $42.6 \pm 2.5$ & $5.4 \pm 0.9$ & $2.1 \pm 1.1$ \\
\hline$>1800$ & 8 & $12.1 \pm 1.5$ & $9.8 \pm 0.7$ & $1.1 \pm 0.4$ & $1.2 \pm 0.6$ \\
\hline
\end{tabular}

Table 3. The number of dimuon events in various invariant mass ranges. The total background is the sum of the events for the SM processes listed. The yields from simulation are normalized relative to the expected cross sections, and overall the simulation is normalized to the observed yield using the number of events in the mass window $60-120 \mathrm{GeV}$, acquired using a prescaled low threshold trigger. Uncertainties include both statistical and systematic components, summed in quadrature.

\section{Statistical analysis, results, and interpretation}

The mass distributions are scrutinized for possible deviations from the SM background predictions. No significant deviations are observed. The limits are expressed as a function of $R_{\sigma}$, which is the ratio of the cross section for dilepton production via a $Z^{\prime}$ boson to the measured cross section for dilepton production via the $\mathrm{Z}$ boson in the mass window 60-120 GeV:

$$
R_{\sigma}=\frac{\sigma\left(\mathrm{pp} \rightarrow \mathrm{Z}^{\prime}+X \rightarrow \ell \ell+X\right)}{\sigma(\mathrm{pp} \rightarrow \mathrm{Z}+X \rightarrow \ell \ell+X)}
$$


Expressing the limits as a ratio, reduces the dependency on the theoretical prediction of the $\mathrm{Z}$ boson cross section as well as the correlated experimental uncertainties.

For the electron and muon channel combination, the branching fractions of these two channels are assumed to be the same. The signal cross section corresponds to that obtained in the narrow width approximation; specifically, off-shell contributions from PDFs and interference effects are not included. The limits are set using a Bayesian method with an unbinned extended likelihood function [13] using the framework developed for statistically combining Higgs boson searches [53], which is based on the RooStats package [54]. The signal probability density function (pdf) used is a convolution of a Breit-Wigner (BW) function and a Gaussian function with exponential tails to either side (Cruijff [55]). The BW function models the intrinsic width of the particle, while the Cruijff function models the detector response. A Crystal Ball (CB) function [56] better describes the signal shape at higher dielectron masses compared to the Cruijff function. Therefore for $m_{\mathrm{ee}}>2300 \mathrm{GeV}$, a CB function is used. The background pdf for the dielectron channel is provided by the following formula:

$$
\begin{array}{ll}
m^{\kappa} \exp \left(\sum_{i=0}^{3} \alpha_{i} m^{i}\right), & \text { if } m \leq 600 \mathrm{GeV} \\
m^{\lambda} \exp \left(\sum_{i=0}^{3} \beta_{i} m^{i}\right), & \text { if } m>600 \mathrm{GeV},
\end{array}
$$

while for the dimuon channel the following functional form is used:

$$
\begin{array}{ll}
m^{\mu} \exp \left(\sum_{i=0}^{2} \gamma_{i} m^{i}\right), & \text { if } m \leq 500 \mathrm{GeV} \\
m^{\nu} \exp \left(\sum_{i=0}^{3} \delta_{i} m^{i}\right), & \text { if } m>500 \mathrm{GeV} .
\end{array}
$$

The different background pdfs for dielectrons and dimuons reflect the different background composition in the two channels. For each final state, the parameters of the background pdf are obtained by fitting the total background distribution produced using SM MC generators and the background arising from misidentified jets deduced from the data. The fits to the background distribution are set for masses above $120 \mathrm{GeV}$.

For the signal cross section we use a positive uniform prior. The systematic uncertainties in the dilepton mass originating from efficiencies, resolution and scale, as discussed in section 4 , are treated as nuisance parameters and assigned log normal priors. The relative mass scale of the different channels is the only uncertainty with a noticeable impact. The limits are calculated in a mass window of \pm 6 times the signal width, with this window being symmetrically enlarged until there is a minimum of 100 data events in it. This procedure sets the level of the statistical uncertainty in the local background amplitude; the level is chosen to dominate the expected systematic uncertainties in the background shape at high mass. The total background uncertainty ranges from approximately $3 \%$ at $200 \mathrm{GeV}$ to $12 \%$ at $5 \mathrm{TeV}$ for both electrons and muons. At low mass it is driven by normalization 

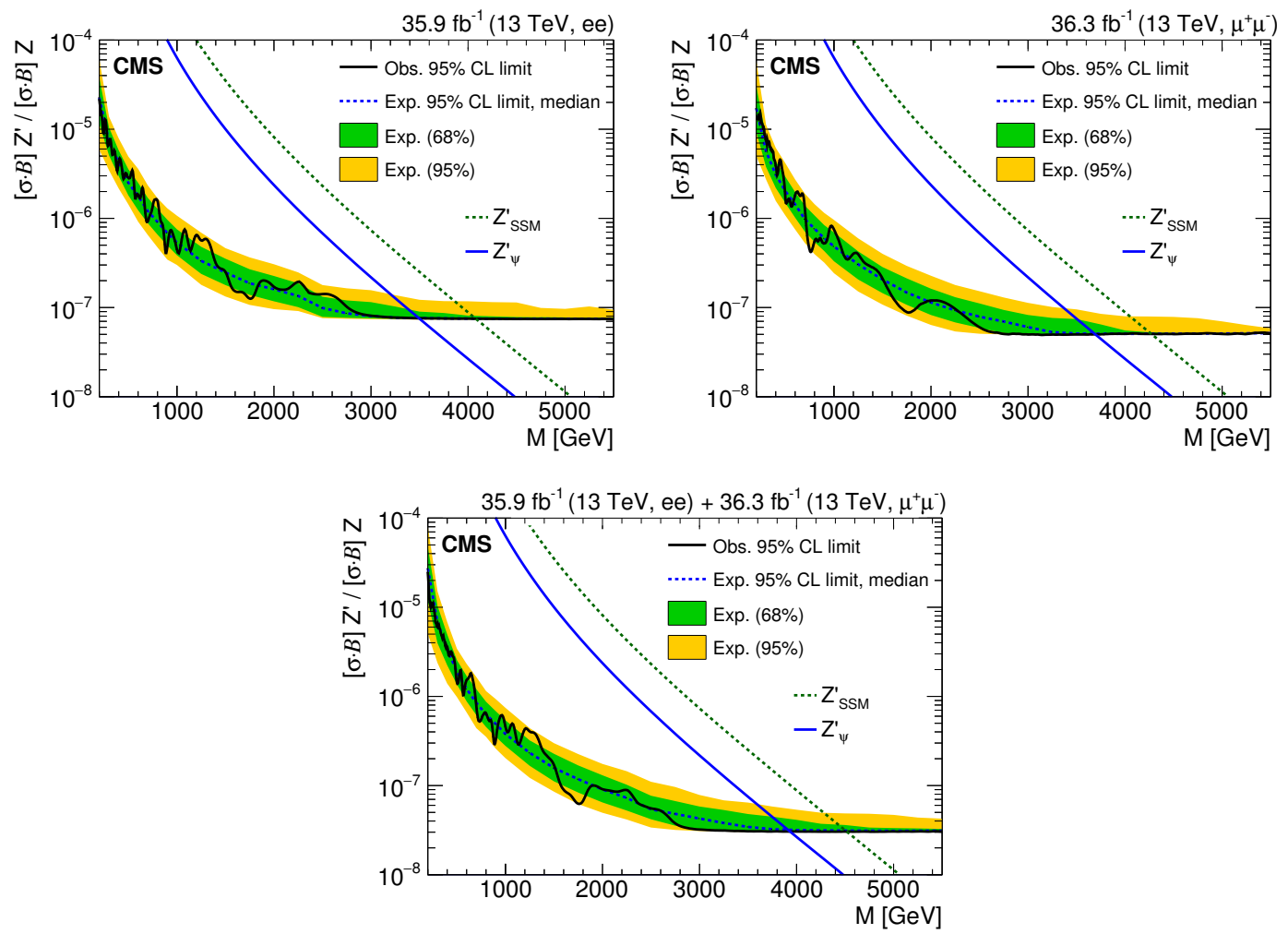

Figure 3. The upper limits at 95\% CL on the product of production cross section and branching fraction for a spin-1 resonance with a width equal to $0.6 \%$ of the resonance mass, relative to the product of production cross section and branching fraction of a $\mathrm{Z}$ boson, for the dielectron channel (left), dimuon channel (right), and their combination (lower). The shaded bands correspond to the 68 and $95 \%$ quantiles for the expected limits. Theoretical predictions for the spin- $1 \mathrm{Z}_{\mathrm{SSM}}^{\prime}$ and $\mathrm{Z}_{\psi}^{\prime}$ resonances are shown for comparison.

uncertainties; while at high mass, PDFs and higher-order corrections are the dominant sources of uncertainty.

The expected and observed limits for a resonance width equal to $0.6 \%$ of the resonance mass are shown in figure 3 for the dielectron channel, dimuon channels, and their combination. Table 4 presents the observed and expected 95\% confidence level (CL) lower limits on the masses of spin- $1 \mathrm{Z}_{\mathrm{SSM}}^{\prime}$ and $\mathrm{Z}_{\psi}^{\prime}$ bosons. Results for widths equal to $0.6,3,5$ and $10 \%$ of the resonance mass are shown in figure 4 for the dielectron channel, dimuon channel, and their combination. For masses below $2 \mathrm{TeV}$ the expected limits become less stringent with increasing resonance widths. At high masses, however, the experimental mass resolution dominates and the limits do not exhibit any dependence on the assumed resonance width. Compared to the default width of $0.6 \%$, an increased width results in less stringent observed and expected limits, and a smoother variation of the observed limit as a function of mass.

The $R_{\sigma}$ curves are shown on the plots to obtain mass limits for $\mathrm{Z}^{\prime}$ signal models. The curves are constructed by dividing the LO cross section of a given model, calculated using the PYTHIA 8.2 program with the NNPDF2.3 [57] PDFs, by the NNLO Z boson cross 

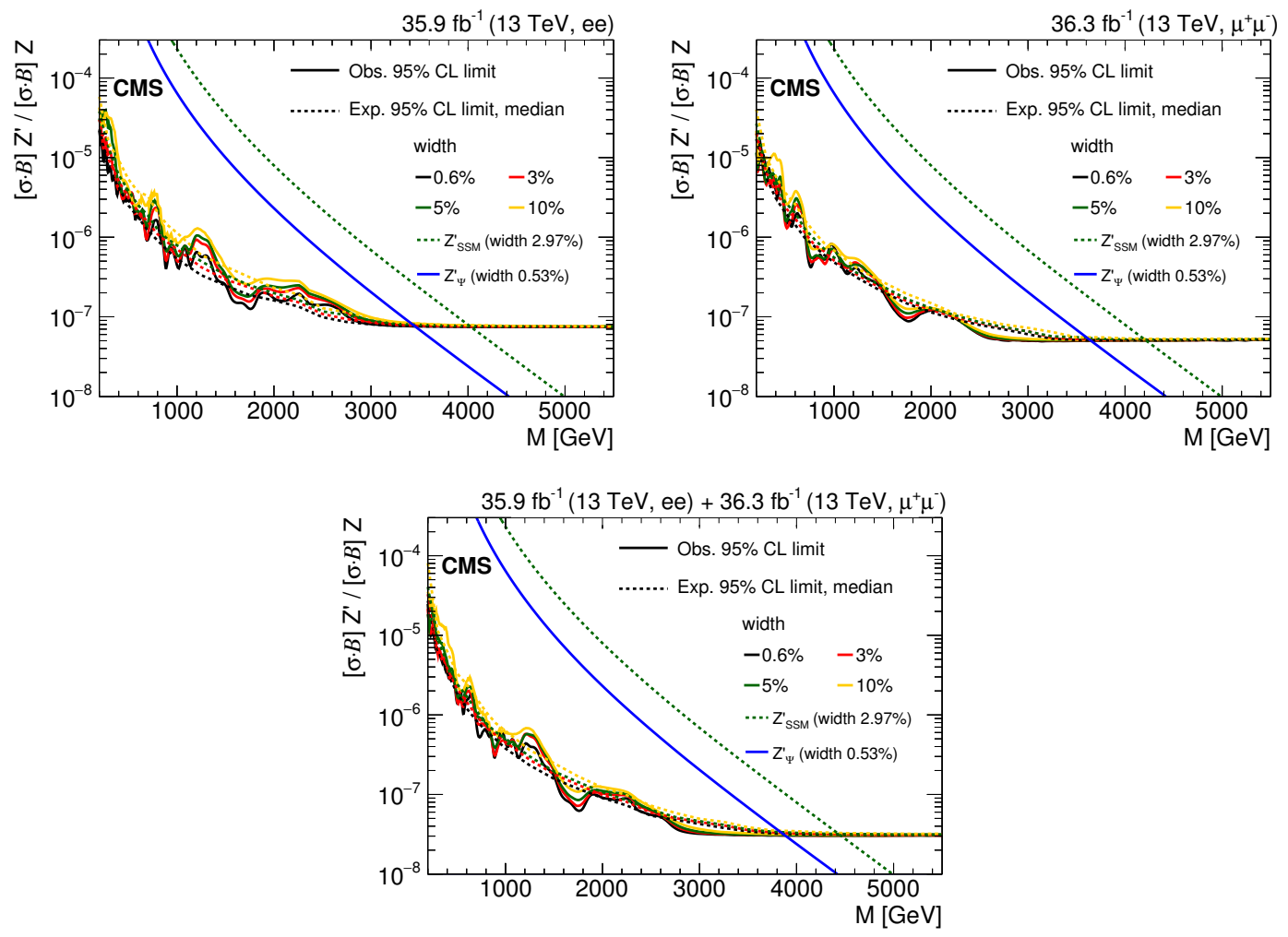

Figure 4. The upper limits at 95\% CL on the product of production cross section and branching fraction for a spin- 1 resonance, for widths equal to $0.6,3,5$, and $10 \%$ of the resonance mass, relative to the product of production cross section and branching fraction for a $\mathrm{Z}$ boson, for the dielectron channel (left), dimuon channel (right), and their combination (lower). Theoretical predictions for the spin-1 $\mathrm{Z}_{\mathrm{SSM}}^{\prime}$ and $\mathrm{Z}_{\psi}^{\prime}$ resonances are also shown.

section of $1928 \pm 73 \mathrm{pb}$ obtained with FEWZ 3.1 [40]. As the limits presented here are set on the on-shell cross section and the PYTHIA event generator includes off-shell effects, the cross section is calculated in a mass window of $\pm 5 \% \sqrt{s}$ centred on the resonance mass, following the prescription of ref. [9]. The validity of this procedure for the $\mathrm{Z}_{\mathrm{SSM}}^{\prime}$ and $\mathrm{Z}_{\psi}^{\prime}$ bosons was explicitly checked in ref. [9] and is found to be accurate to approximately $5-7 \%$. To account for NNLO QCD effects, the LO cross sections are multiplied by a mass independent $K$ factor. The value of the $K$-factor is estimated at a dilepton mass of $4.5 \mathrm{TeV}$ and found to be consistent with unity. Applying a mass dependent $K$-factor, the $\mathrm{Z}_{\psi}^{\prime}$ resonance mass limit differs by only $50 \mathrm{GeV}$, justifying the use of the simpler mass independent $K$-factor.

For the $\mathrm{Z}_{\mathrm{SSM}}^{\prime}$ and $\mathrm{Z}_{\psi}^{\prime}$ bosons, we obtain $95 \%$ CL lower mass limits of 4.50 and $3.90 \mathrm{TeV}$, respectively. Recent measurements from the ATLAS experiment, based on $36.1 \mathrm{fb}^{-1}$ of proton-proton collision data collected at $\sqrt{s}=13 \mathrm{TeV}$ in 2015 and 2016 [18], have obtained 95\% CL lower mass limits of 4.5 and $3.8 \mathrm{TeV}$ for the $\mathrm{Z}_{\mathrm{SSM}}^{\prime}$ and $\mathrm{Z}_{\psi}^{\prime}$ bosons, respectively.

In figure 5 , the cross section limit curve from figure 3 is translated into the $\left(c_{\mathrm{d}}, c_{\mathrm{u}}\right)$ plane. The LO cross section is a linear function of $c_{\mathrm{d}}$ and $c_{\mathrm{u}}\left(\sigma_{\mathrm{LO}} \propto c_{\mathrm{d}} w_{\mathrm{d}}+c_{\mathrm{u}} w_{\mathrm{u}}\right.$, where $w_{\mathrm{d}} / w_{\mathrm{u}}$ is in the range $0.5-0.6$ for the results shown here) and hence a single value of the 


\begin{tabular}{|ccccc|}
\hline \multirow{2}{*}{ Channel } & \multicolumn{2}{c}{$\mathrm{Z}_{\text {SSM }}^{\prime}$} & \multicolumn{2}{c|}{$\mathrm{Z}_{\psi}^{\prime}$} \\
& Obs. $[\mathrm{TeV}]$ & Exp. $[\mathrm{TeV}]$ & Obs. $[\mathrm{TeV}]$ & Exp. $[\mathrm{TeV}]$ \\
\hline ee & 4.10 & 4.10 & 3.45 & 3.45 \\
$\mu^{+} \mu^{-}$ & 4.25 & 4.25 & 3.70 & 3.70 \\
$\mathrm{ee}+\mu^{+} \mu^{-}$ & 4.50 & 4.50 & 3.90 & 3.90 \\
\hline
\end{tabular}

Table 4. The observed and expected $95 \%$ CL lower limits on the masses of spin- $1 \mathrm{Z}_{\mathrm{SSM}}^{\prime}$ and $\mathrm{Z}_{\psi}^{\prime}$ bosons, assuming a signal width of $0.6 \%(3.0 \%)$ of the resonance mass for $\mathrm{Z}_{\psi}^{\prime}\left(\mathrm{Z}_{\mathrm{SSM}}^{\prime}\right)$.

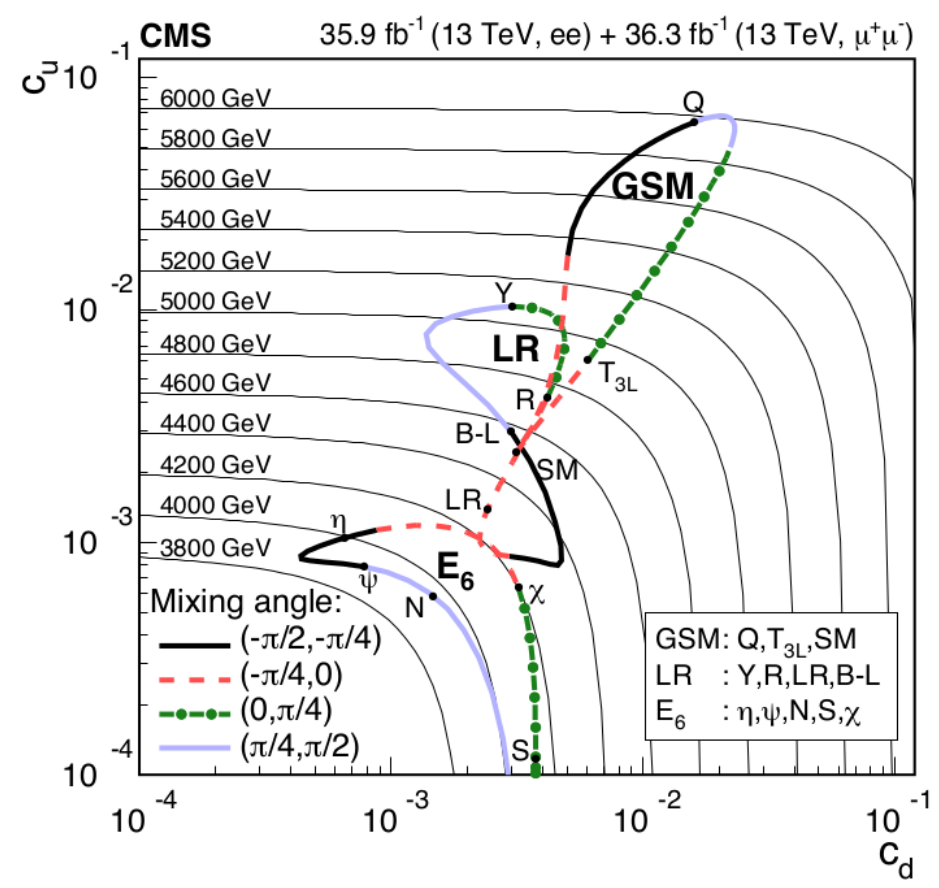

Figure 5. Limits in the $\left(c_{\mathrm{d}}, c_{\mathrm{u}}\right)$ plane obtained by recasting the combined limit at $95 \% \mathrm{CL}$ on the $\mathrm{Z}^{\prime}$ boson cross section from dielectron and dimuon channels. For a given $\mathrm{Z}^{\prime}$ boson mass, the cross section limit results in a solid thin black line. These lines are labelled with the relevant $\mathrm{Z}^{\prime}$ boson masses. The closed contours representing the GSM, LR, and $\mathrm{E}_{6}$ model classes are composed of thick line segments. Each point on a segment corresponds to a particular model, and the location of the point gives the mass limit on the relevant $\mathrm{Z}^{\prime}$ boson. As indicated in the bottom left legend, the segment line styles correspond to ranges of the particular mixing angle for each considered model. The bottom right legend indicates the constituents of each model class.

cross section is represented by a straight line in the $\left(c_{\mathrm{d}}, c_{\mathrm{u}}\right)$ plane. In the log-log plot shown in figure 5 , the thin lines labelled with a mass value correspond to the cross section limit at that mass. The closed contours representing the GSM, LR, and $\mathrm{E}_{6}$ model classes are composed of thick line segments which correspond to ranges of the particular mixing angle for each considered model. A brief description of the models is given in section 1 with further information provided in table 1 and the exact definition of the models discussed in ref. [6]. The mass limit on the relevant $\mathrm{Z}^{\prime}$ boson in any model, where $c_{\mathrm{d}}$ and $c_{\mathrm{u}}$ have been determined, can be read off this plot. 

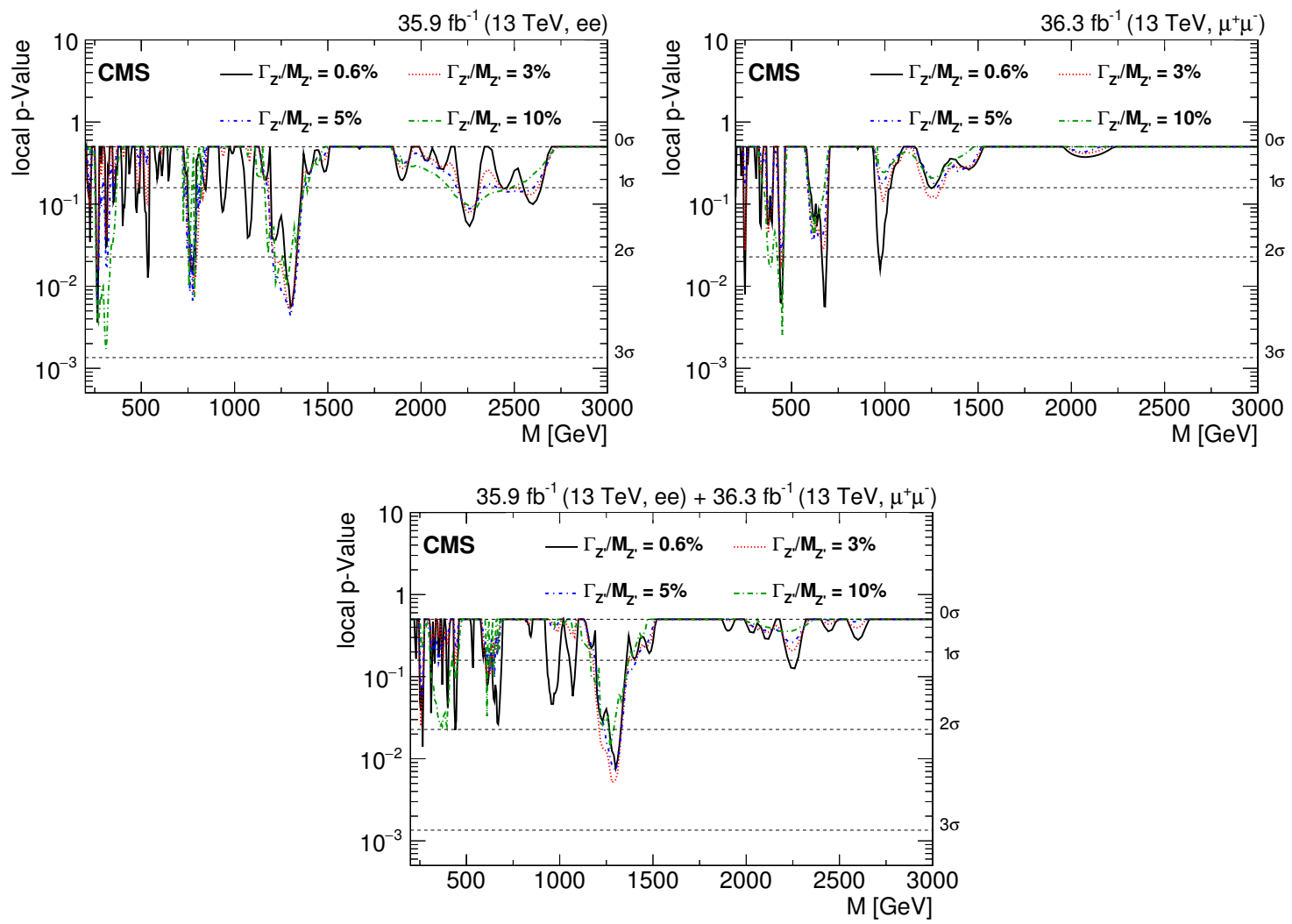

Figure 6. The observed local $p$-value for the dielectron channel (left), dimuon channel (right), and their combination (lower) as a function of the dilepton invariant mass.

For completeness we quantify a possible presence of an excess of events over what is expected for the background by computing the $p$-value. The $p$-value for different signal width hypotheses is shown for both the separate and combined channels in figure 6 . The largest excess in the combined result is observed around $M=1300 \mathrm{GeV}$ having a local significance of around 2.5 s.d. for a spin-1 resonance with widths 0.6 to $5.0 \%$. This corresponds to a global significance of -0.92 s.d. after taking into consideration the look elsewhere effect [58] in the mass range 200 to $5500 \mathrm{GeV}$. The global significance is expressed as the corresponding number of standard deviations using the one-sided Gaussian tail convention. The methodology of the $p$-value computation is described in ref. [59].

The expected and observed limits for a spin-2 resonance with intrinsic widths of 0.01 , 0.36 , and $1.42 \mathrm{GeV}$ corresponding to coupling parameters $k / \bar{M}_{\mathrm{Pl}}$ of $0.01,0.05$, and 0.10 , are shown in figure 7 for the dielectron channel, dimuon channel, and their combination. Table 5 presents the values of the observed and expected 95\% CL lower limits of the aforementioned models. The signal production cross sections, calculated using the PYTHIA 8.2 program with the NNPDF2.3 PDFs at LO, are multiplied by a $K$-factor of 1.6 to account for NLO effects [60]. The PI contribution to the production cross sections is small enough to be ignored.

The results are also interpreted in the context of a simplified model with a DM particle that has sizeable interactions with SM fermions through an additional spin-1 high-mass par- 

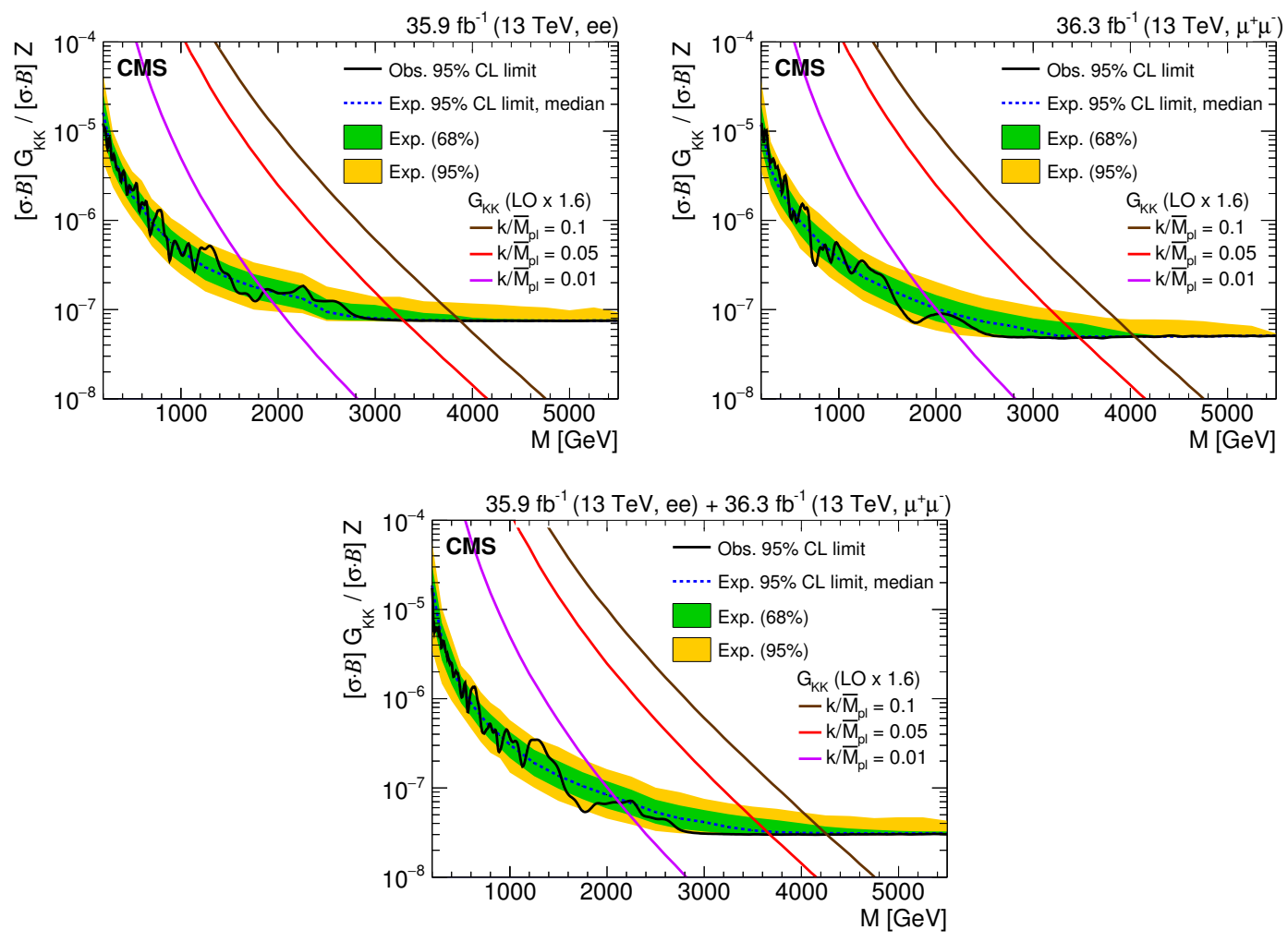

Figure 7. The upper limits at $95 \% \mathrm{CL}$ on the product of production cross section and branching fraction for a spin-2 resonance, relative to the product of production cross section and branching fraction of a $\mathrm{Z}$ boson, for the dielectron channel (left), dimuon channel (right), and their combination (lower). The shaded bands correspond to the 68 and $95 \%$ quantiles for the expected limits. Theoretical predictions for the spin- 2 resonances with widths equal to $0.01,0.36$, and $1.42 \mathrm{GeV}$ corresponding to coupling parameters $k / \bar{M}_{\mathrm{Pl}}$ of $0.01,0.05$, and 0.10 are shown for comparison.

\begin{tabular}{|ccccccc|}
\hline \multirow{2}{*}{ Channel } & \multicolumn{2}{c}{$k / \bar{M}_{\mathrm{Pl}}=0.01$} & \multicolumn{2}{c|}{$k / \bar{M}_{\mathrm{Pl}}=0.05$} & \multicolumn{2}{c|}{$k / \bar{M}_{\mathrm{Pl}}=0.1$} \\
& Obs. $[\mathrm{TeV}]$ & Exp. $[\mathrm{TeV}]$ & Obs. $[\mathrm{TeV}]$ & Exp. $[\mathrm{TeV}]$ & Obs. [TeV] & Exp. [TeV] \\
\hline ee & 1.85 & 1.85 & 3.30 & 3.30 & 3.90 & 3.90 \\
$\mu^{+} \mu^{-}$ & 2.05 & 2.00 & 3.50 & 3.50 & 4.05 & 4.05 \\
$\mathrm{ee}+\mu^{+} \mu^{-}$ & 2.10 & 2.05 & 3.65 & 3.60 & 4.25 & 4.25 \\
\hline
\end{tabular}

Table 5. The observed and expected 95\% CL lower limits on the masses of spin-2 resonances with widths equal to $0.01,0.36$ and $1.42 \mathrm{GeV}$ corresponding to coupling parameters $k / \bar{M}_{\mathrm{Pl}}$ of $0.01,0.05$, and 0.10 .

ticle mediating the SM-DM interaction. In the simplified model under consideration [26], only one DM particle exists, which is assumed to be a Dirac fermion. Limits are presented in figure 8 for two cases with different sets of benchmark coupling values. The first case corresponds to a vector mediator with small couplings to leptons while the second one corresponds to an axial-vector mediator with equal couplings to quarks and leptons. The cross sections for lepton production are calculated at NLO in QCD, using the DMsimP implementation [27] of the simplified model in MADGRAPH5_aMC@NLO version 2.5.2 [46]. 

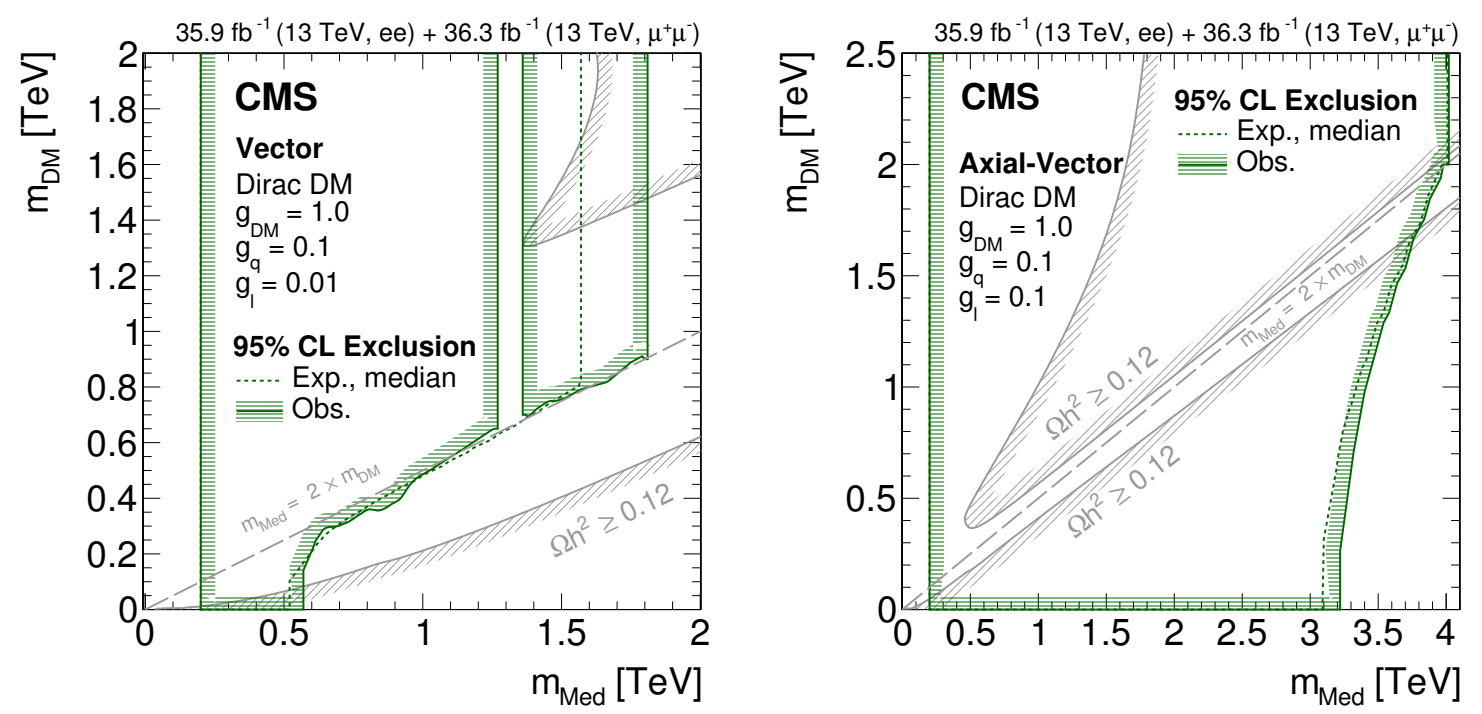

Figure 8. Limits at $95 \%$ confidence level for the masses of the DM particle, which is assumed to be Dirac fermion, and its associated mediator, in a simplified model of DM production via a vector (left) or axial vector (right) mediator. The parameter exclusion is obtained by comparing the limits on product of the production cross section and the branching fraction for decay to a $\mathrm{Z}$ boson, with the values obtained from calculations in the simplified model. For each combination of the DM particle and mediator mass values, the width of the mediator is taken into account in the limit calculation. The lines with the hatching represents the excluded regions. The solid grey lines, marked as " $\Omega h^{2} \geq 0.12$ ", correspond to parameter regions that reproduce the observed DM relic density in the universe $[4,26,62,63]$, with the hatched area indicating the region where the DM relic abundance exceeds the observed value.

Assuming the optimistic axial-vector coupling scenario and $m_{\mathrm{DM}}>m_{\mathrm{Med}} / 2$, the signal cross section for the production of an electron or muon pair within the analysis acceptance ranges between approximately $100 \mathrm{pb}$ at low values of the mediator mass (around $200 \mathrm{GeV}$ ), and $0.1 \mathrm{fb}$ for higher values (around $4 \mathrm{TeV}$ ). The partial and total mediator decay widths, calculated at LO in QCD, are included via the MADWIDTH package [61].

While the DM particle is not probed directly, its mass indirectly modulates the sensitivity of the dilepton search. For low values of the DM particle mass $m_{\mathrm{DM}}<m_{\mathrm{Med}} / 2$, the mediator boson will dominantly decay into DM particles, thus reducing the branching fraction to leptons, and making the mediator harder to probe in this search. At high values of the DM particle mass $m_{\mathrm{DM}}>m_{\mathrm{Med}} / 2$, the mediator cannot decay to the DM particles and the leptonic branching fraction becomes sizeable. In the vector mediator model, the relatively small leptonic couplings mostly limit the sensitivity of this analysis to the regime of $m_{\mathrm{DM}}>m_{\mathrm{Med}} / 2$. This regime is especially interesting to probe since it is almost inaccessible to typical searches based on missing transverse momentum [64]. In the axial-vector mediator model, the leptonic couplings of the mediator are sizeable and an exclusion is also possible for $m_{\mathrm{DM}}<m_{\mathrm{Med}} / 2$. In the former case, the limit on the mediator mass reaches up to $1.8 \mathrm{TeV}$, depending on the mass of the DM particle; while in the latter case the limit reaches the 3.0-4.0 TeV, depending again on the mass of the DM particle. In the vector 
mediator model, the observed exclusion reaches up to values of the mediator mass equal to approximately $1.8(0.6) \mathrm{TeV}$ above (below) the diagonal, $m_{\mathrm{DM}}=m_{\mathrm{Med}} / 2$. In the region where the mediator mass is equal to approximately $1.3 \mathrm{TeV}$, an upward fluctuation in the data (figure 3) results in a small above-diagonal region that is not excluded. Assuming that there is no new physics other than the mediator and DM particle, the relic density of DM in the universe, $\Omega h^{2}$, can be calculated. Regions of parameter space that reproduce the observed value $\Omega h^{2} \approx 0.12$ [4], are indicated in figure 8. Numerical values are obtained from ref. [26], where they were calculated using MADDM in version 2.06 [62, 63]. In the hatched area, the DM would be overabundant in the universe. However, it is unlikely that new physics is fully described by the simplified model considered here, and the relic density constraint is not a stringent constraint, as additional new phenomena may modify its calculated value.

\section{Summary}

A search for narrow resonances in dielectron and dimuon invariant mass spectra has been performed using data recorded in 2016 from proton-proton collisions at $\sqrt{s}=13 \mathrm{TeV}$. The integrated luminosity for the dielectron sample is $35.9 \mathrm{fb}^{-1}$ and for the dimuon sample is $36.3 \mathrm{fb}^{-1}$. Observations are in agreement with standard model expectations. Upper limits at $95 \%$ confidence level on the product of a narrow-resonance production cross section and branching fraction to dileptons have been calculated in a model-independent manner to enable interpretation in the framework of models predicting a narrow dielectron or dimuon resonance. A scan of different intrinsic width hypotheses is performed.

Limits are set on the masses of various hypothetical particles. For the $\mathrm{Z}_{\mathrm{SSM}}^{\prime}$ particle, which arises in the sequential standard model, and for the superstring-inspired $Z_{\psi}^{\prime}$ particle, 95\% confidence level lower mass limits for the combined channels are found to be 4.50 and $3.90 \mathrm{TeV}$, respectively. These limits extend the previous ones from CMS by $1.1 \mathrm{TeV}$ in both models. The corresponding limits for Kaluza-Klein gravitons arising in the RandallSundrum model of extra dimensions with coupling parameters $k / \bar{M}_{\mathrm{Pl}}$ of $0.01,0.05$, and 0.10 are $2.10,3.65$, and $4.25 \mathrm{TeV}$, respectively. The limits extend previous published CMS results by $0.6(1.1) \mathrm{TeV}$ for a $k / \bar{M}_{\mathrm{Pl}}$ value of $0.01(0.10)$. Finally, limits at $95 \%$ confidence level are obtained for the masses of the dark matter particle and its associated mediator, in a simplified model of dark matter production via a vector or axial vector mediator.

\section{Acknowledgments}

We congratulate our colleagues in the CERN accelerator departments for the excellent performance of the LHC and thank the technical and administrative staffs at CERN and at other CMS institutes for their contributions to the success of the CMS effort. In addition, we gratefully acknowledge the computing centres and personnel of the Worldwide LHC Computing Grid for delivering so effectively the computing infrastructure essential to our analyses. Finally, we acknowledge the enduring support for the construction and operation of the LHC and the CMS detector provided by the following funding agencies: BMWFW and FWF (Austria); FNRS and FWO (Belgium); CNPq, CAPES, FAPERJ, 
and FAPESP (Brazil); MES (Bulgaria); CERN; CAS, MoST, and NSFC (China); COLCIENCIAS (Colombia); MSES and CSF (Croatia); RPF (Cyprus); SENESCYT (Ecuador); MoER, ERC IUT, and ERDF (Estonia); Academy of Finland, MEC, and HIP (Finland); CEA and CNRS/IN2P3 (France); BMBF, DFG, and HGF (Germany); GSRT (Greece); NKFIA (Hungary); DAE and DST (India); IPM (Iran); SFI (Ireland); INFN (Italy); MSIP and NRF (Republic of Korea); LAS (Lithuania); MOE and UM (Malaysia); BUAP, CINVESTAV, CONACYT, LNS, SEP, and UASLP-FAI (Mexico); MBIE (New Zealand); PAEC (Pakistan); MSHE and NSC (Poland); FCT (Portugal); JINR (Dubna); MON, RosAtom, RAS, RFBR and RAEP (Russia); MESTD (Serbia); SEIDI, CPAN, PCTI and FEDER (Spain); Swiss Funding Agencies (Switzerland); MST (Taipei); ThEPCenter, IPST, STAR, and NSTDA (Thailand); TUBITAK and TAEK (Turkey); NASU and SFFR (Ukraine); STFC (United Kingdom); DOE and NSF (U.S.A.).

Individuals have received support from the Marie-Curie programme and the European Research Council and Horizon 2020 Grant, contract No. 675440 (European Union); the Leventis Foundation; the A. P. Sloan Foundation; the Alexander von Humboldt Foundation; the Belgian Federal Science Policy Office; the Fonds pour la Formation à la Recherche dans l'Industrie et dans l'Agriculture (FRIA-Belgium); the Agentschap voor Innovatie door Wetenschap en Technologie (IWT-Belgium); the F.R.S.-FNRS and FWO (Belgium) under the "Excellence of Science - EOS" - be.h project n. 30820817; the Ministry of Education, Youth and Sports (MEYS) of the Czech Republic; the Lendület ("Momentum") Programme and the János Bolyai Research Scholarship of the Hungarian Academy of Sciences, the New National Excellence Program ÚNKP, the NKFIA research grants 123842, 123959, 124845, 124850 and 125105 (Hungary); the Council of Science and Industrial Research, India; the HOMING PLUS programme of the Foundation for Polish Science, cofinanced from European Union, Regional Development Fund, the Mobility Plus programme of the Ministry of Science and Higher Education, the National Science Center (Poland), contracts Harmonia 2014/14/M/ST2/00428, Opus 2014/13/B/ST2/02543, 2014/15/B/ST2/03998, and 2015/19/B/ST2/02861, Sonata-bis 2012/07/E/ST2/01406; the National Priorities Research Program by Qatar National Research Fund; the Programa Estatal de Fomento de la Investigación Científica y Técnica de Excelencia María de Maeztu, grant MDM-2015-0509 and the Programa Severo Ochoa del Principado de Asturias; the Thalis and Aristeia programmes cofinanced by EU-ESF and the Greek NSRF; the Rachadapisek Sompot Fund for Postdoctoral Fellowship, Chulalongkorn University and the Chulalongkorn Academic into Its 2nd Century Project Advancement Project (Thailand); the Welch Foundation, contract C-1845; and the Weston Havens Foundation (U.S.A.).

Open Access. This article is distributed under the terms of the Creative Commons Attribution License (CC-BY 4.0), which permits any use, distribution and reproduction in any medium, provided the original author(s) and source are credited.

\section{References}

[1] A. Leike, The Phenomenology of extra neutral gauge bosons, Phys. Rept. 317 (1999) 143 [hep-ph/9805494] [INSPIRE]. 
[2] P. Langacker, The physics of heavy $Z^{\prime}$ gauge bosons, Rev. Mod. Phys. 81 (2009) 1199 [arXiv:0801.1345] [INSPIRE].

[3] L. Randall and R. Sundrum, A large mass hierarchy from a small extra dimension, Phys. Rev. Lett. 83 (1999) 3370 [hep-ph/9905221] [INSPIRE].

[4] Planck collaboration, P.A.R. Ade et al., Planck 2015 results. XIII. Cosmological parameters, Astron. Astrophys. 594 (2016) A13 [arXiv:1502.01589] [INSPIRE].

[5] M. Carena, A. Daleo, B.A. Dobrescu and T.M.P. Tait, $Z^{\prime}$ gauge bosons at the Tevatron, Phys. Rev. D 70 (2004) 093009 [hep-ph/0408098] [INSPIRE].

[6] E. Accomando et al., Z' physics with early LHC data, Phys. Rev. D 83 (2011) 075012 [arXiv: 1010.6058] [INSPIRE].

[7] G. Altarelli, B. Mele and M. Ruiz-Altaba, Searching for new heavy vector bosons in $p \bar{p}$ colliders, Z. Phys. C 45 (1989) 109 [Erratum ibid. C 47 (1990) 676] [INSPIRE].

[8] J.L. Hewett and T.G. Rizzo, Low-energy phenomenology of superstring inspired $E_{6}$ models, Phys. Rept. 183 (1989) 193 [INSPIRE].

[9] E. Accomando et al., $Z^{\prime}$ at the LHC: interference and finite width effects in Drell-Yan, JHEP 10 (2013) 153 [arXiv: 1304.6700] [INSPIRE].

[10] CMS collaboration, Search for resonances in the dilepton mass distribution in pp collisions at $\sqrt{s}=7 \mathrm{TeV}$, JHEP 05 (2011) 093 [arXiv: 1103.0981] [INSPIRE].

[11] CMS collaboration, Search for narrow resonances in dilepton mass spectra in pp collisions at $\sqrt{s}=7 \mathrm{TeV}$, Phys. Lett. B 714 (2012) 158 [arXiv:1206.1849] [inSPIRE].

[12] CMS collaboration, Search for heavy narrow dilepton resonances in pp collisions at $\sqrt{s}=7$ $\mathrm{TeV}$ and $\sqrt{s}=8 \mathrm{TeV}$, Phys. Lett. B 720 (2013) 63 [arXiv:1212.6175] [INSPIRE].

[13] CMS collaboration, Search for physics beyond the standard model in dilepton mass spectra in proton-proton collisions at $\sqrt{s}=8 \mathrm{TeV}$, JHEP 04 (2015) 025 [arXiv:1412.6302] [INSPIRE].

[14] CMS collaboration, Search for narrow resonances in dilepton mass spectra in proton-proton collisions at $\sqrt{s}=13 \mathrm{TeV}$ and combination with $8 \mathrm{TeV}$ data, Phys. Lett. B 768 (2017) 57 [arXiv: 1609.05391] [INSPIRE].

[15] ATLAS collaboration, Search for high mass dilepton resonances in pp collisions at $\sqrt{s}=7$ TeV with the ATLAS experiment, Phys. Lett. B 700 (2011) 163 [arXiv:1103.6218] [INSPIRE].

[16] ATLAS collaboration, Search for high-mass resonances decaying to dilepton final states in pp collisions at $\sqrt{s}=7 \mathrm{TeV}$ with the ATLAS detector, JHEP 11 (2012) 138 [arXiv: 1209.2535] [INSPIRE].

[17] ATLAS collaboration, Search for high-mass dilepton resonances in pp collisions at $\sqrt{s}=8$ TeV with the ATLAS detector, Phys. Rev. D 90 (2014) 052005 [arXiv: 1405.4123] [InSPIRE].

[18] ATLAS collaboration, Search for new high-mass phenomena in the dilepton final state using $36 \mathrm{fb}^{-1}$ of proton-proton collision data at $\sqrt{\mathrm{s}}=13 \mathrm{TeV}$ with the ATLAS detector, JHEP 10 (2017) 182 [arXiv: 1707.02424] [INSPIRE].

[19] L. Randall and R. Sundrum, An alternative to compactification, Phys. Rev. Lett. 83 (1999) 4690 [hep-th/9906064] [INSPIRE].

[20] CDF collaboration, T. Aaltonen et al., Search for high-mass $e^{+} e^{-}$resonances in $p \bar{p}$ collisions at $\sqrt{s}=1.96 \mathrm{TeV}$, Phys. Rev. Lett. 102 (2009) 031801 [arXiv:0810.2059] [INSPIRE]. 
[21] CDF collaboration, T. Aaltonen et al., A search for high-mass resonances decaying to dimuons at CDF, Phys. Rev. Lett. 102 (2009) 091805 [arXiv:0811.0053] [InSPIRE].

[22] D0 collaboration, V.M. Abazov et al., Search for Randall-Sundrum gravitons in the dielectron and diphoton final states with $5.4 \mathrm{fb}^{-1}$ of data from $p \bar{p}$ collisions at $\sqrt{s}=1.96$ TeV, Phys. Rev. Lett. 104 (2010) 241802 [arXiv:1004.1826] [INSPIRE].

[23] D0 collaboration, V.M. Abazov et al., Search for a heavy neutral gauge boson in the dielectron channel with $5.4 \mathrm{fb}^{-1}$ of $p \bar{p}$ collisions at $\sqrt{s}=1.96 \mathrm{TeV}$, Phys. Lett. B 695 (2011) 88 [arXiv: 1008.2023] [INSPIRE].

[24] CDF collaboration, T. Aaltonen et al., Search for high mass resonances decaying to muon pairs in $\sqrt{s}=1.96$ TeV pp collisions, Phys. Rev. Lett. 106 (2011) 121801 [arXiv:1101.4578] [INSPIRE].

[25] CDF collaboration, T. Aaltonen et al., Search for new dielectron resonances and Randall-Sundrum gravitons at the collider detector at Fermilab, Phys. Rev. Lett. 107 (2011) 051801 [arXiv: 1103.4650] [INSPIRE].

[26] A. Albert et al., Recommendations of the LHC dark matter working group: comparing LHC searches for heavy mediators of dark matter production in visible and invisible decay channels, arXiv:1703.05703 [INSPIRE].

[27] M. Backović et al., Higher-order QCD predictions for dark matter production at the LHC in simplified models with s-channel mediators, Eur. Phys. J. C 75 (2015) 482 [arXiv: 1508.05327] [INSPIRE].

[28] C. Battilana, The CMS muon system: status and upgrades for LHC Run-2 and performance of muon reconstruction with 13 TeV data, 2017 JINST 12 C01048.

[29] CMS collaboration, The CMS experiment at the CERN LHC, 2008 JINST 3 S08004 [INSPIRE].

[30] CMS collaboration, The CMS trigger system, 2017 JINST 12 P01020 [arXiv:1609.02366] [INSPIRE].

[31] P. Nason, A new method for combining NLO QCD with shower Monte Carlo algorithms, JHEP 11 (2004) 040 [hep-ph/0409146] [INSPIRE].

[32] S. Frixione, P. Nason and C. Oleari, Matching NLO QCD computations with Parton Shower simulations: the POWHEG method, JHEP 11 (2007) 070 [arXiv:0709.2092] [INSPIRE].

[33] S. Alioli et al., A general framework for implementing NLO calculations in shower Monte Carlo programs: the POWHEG BOX, JHEP 06 (2010) 043 [arXiv: 1002.2581] [INSPIRE].

[34] S. Alioli, P. Nason, C. Oleari and E. Re, NLO vector-boson production matched with shower in POWHEG, JHEP 07 (2008) 060 [arXiv:0805.4802] [INSPIRE].

[35] S. Frixione, P. Nason and G. Ridolfi, A Positive-weight next-to-leading-order Monte Carlo for heavy flavour hadroproduction, JHEP 09 (2007) 126 [arXiv:0707.3088] [INSPIRE].

[36] E. Re, Single-top Wt-channel production matched with parton showers using the POWHEG method, Eur. Phys. J. C 71 (2011) 1547 [arXiv:1009.2450] [INSPIRE].

[37] NNPDF collaboration, R.D. Ball et al., Parton distributions for the LHC Run II, JHEP 04 (2015) 040 [arXiv: 1410.8849] [INSPIRE].

[38] T. Sjöstrand et al., An introduction to PYTHIA 8.2, Comput. Phys. Commun. 191 (2015) 159 [arXiv:1410.3012] [INSPIRE]. 
[39] CMS collaboration, Event generator tunes obtained from underlying event and multiparton scattering measurements, Eur. Phys. J. C 76 (2016) 155 [arXiv:1512.00815] [InSPIRE].

[40] Y. Li and F. Petriello, Combining QCD and electroweak corrections to dilepton production in FEWZ, Phys. Rev. D 86 (2012) 094034 [arXiv: 1208.5967] [INSPIRE].

[41] A. Manohar, P. Nason, G.P. Salam and G. Zanderighi, How bright is the proton? A precise determination of the photon parton distribution function, Phys. Rev. Lett. 117 (2016) 242002 [arXiv: 1607.04266] [INSPIRE].

[42] J. Butterworth et al., PDF4LHC recommendations for LHC Run II, J. Phys. G 43 (2016) 023001 [arXiv: 1510.03865] [INSPIRE].

[43] D. Bourilkov, Photon-induced background for dilepton searches and measurements in pp collisions at $13 \mathrm{TeV}$, arXiv:1606.00523 [INSPIRE].

[44] D. Bourilkov, Exploring the LHC landscape with dileptons, arXiv:1609.08994 [INSPIRE].

[45] M. Czakon and A. Mitov, Top++: a program for the calculation of the top-pair cross-section at hadron colliders, Comput. Phys. Commun. 185 (2014) 2930 [arXiv:1112.5675] [INSPIRE].

[46] J. Alwall et al., The automated computation of tree-level and next-to-leading order differential cross sections and their matching to parton shower simulations, JHEP 07 (2014) 079 [arXiv: 1405.0301] [INSPIRE].

[47] M.R. Whalley, D. Bourilkov and R.C. Group, The Les Houches accord PDFs (LHAPDF) and LHAGLUE, in HERA and the LHC: a workshop on the implications of HERA for LHC physics. Proceedings, Part B, hep-ph/0508110 [INSPIRE].

[48] D. Bourilkov, R.C. Group and M.R. Whalley, LHAPDF: PDF use from the Tevatron to the $L H C$, talk given at the TeV4LHC Workshop - $4^{\text {th }}$ meeting, October 20-22, Batavia, Illinois U.S.A. (2006), hep-ph/0605240 [INSPIRE].

[49] A. Buckley et al., LHAPDF6: parton density access in the LHC precision era, Eur. Phys. J. C 75 (2015) 132 [arXiv: 1412.7420] [INSPIRE].

[50] GEANT4 collaboration, S. Agostinelli et al., GEANT4 - a simulation toolkit, Nucl. Instrum. Meth. A 506 (2003) 250 [inSPIRE].

[51] CMS collaboration, Performance of electron reconstruction and selection with the CMS detector in proton-proton collisions at $\sqrt{s}=8 \mathrm{TeV}, 2015$ JINST $10 \mathrm{P} 06005$ [arXiv: 1502.02701] [INSPIRE].

[52] CMS collaboration, Performance of CMS muon reconstruction in pp collision events at $\sqrt{s}=7 \mathrm{TeV}, 2012 \operatorname{JINST} 7 \mathrm{P} 10002$ [arXiv:1206.4071] [INSPIRE].

[53] ATLAS and CMS Collaborations and The LHC Higgs Combination Group, Procedure for the LHC Higgs boson search combination in Summer 2011, CMS-NOTE-2011-005 (2011).

[54] L. Moneta et al., The RooStats project, PoS(ACAT2010) 057 [arXiv: 1009.1003] [INSPIRE].

[55] BABar collaboration, P. del Amo Sanchez et al., Study of $B \rightarrow X \gamma$ decays and determination of $\left|V_{t d} / V_{t s}\right|$, Phys. Rev. D 82 (2010) 051101 [arXiv:1005.4087] [INSPIRE].

[56] M.J. Oreglia, A study of the reactions $\psi^{\prime} \rightarrow \gamma \gamma \psi$, Ph.D. thesis, Stanford University, U.S.A. (1980) [SLAC-R-236].

[57] R.D. Ball et al., Parton distributions with LHC data, Nucl. Phys. B 867 (2013) 244 [arXiv: 1207.1303] [INSPIRE]. 
[58] E. Gross and O. Vitells, Trial factors for the look elsewhere effect in high energy physics, Eur. Phys. J. C 70 (2010) 525 [arXiv: 1005.1891] [INSPIRE].

[59] CMS collaboration, Observation of a new boson at a mass of $125 \mathrm{GeV}$ with the CMS experiment at the LHC, Phys. Lett. B 716 (2012) 30 [arXiv:1207.7235] [INSPIRE].

[60] P. Mathews, V. Ravindran and K. Sridhar, NLO-QCD corrections to dilepton production in the Randall-Sundrum model, JHEP 10 (2005) 031 [hep-ph/0506158] [INSPIRE].

[61] J. Alwall et al., Computing decay rates for new physics theories with FeynRules and MadGraph 5_aMC@NLO, Comput.Phys.Commun. 197 (2015) 312 [arXiv:1402.1178] [INSPIRE].

[62] M. Backović et al., MadDM: new dark matter tool in the LHC era, AIP Conf. Proc. 1743 (2016) 060001 [arXiv: 1509.03683] [INSPIRE].

[63] M. Backovic, K. Kong and M. McCaskey, MadDM v.1.0: computation of dark matter relic abundance using MadGraph5, Physics of the Dark Universe 5-6 (2014) 18 [arXiv: 1308.4955] [INSPIRE].

[64] CMS collaboration, Search for dark matter produced with an energetic jet or a hadronically decaying $W$ or $Z$ boson at $\sqrt{s}=13 \mathrm{TeV}$, JHEP 07 (2017) 014 [arXiv:1703.01651] [INSPIRE]. 


\section{The CMS collaboration}

\section{Yerevan Physics Institute, Yerevan, Armenia}

A.M. Sirunyan, A. Tumasyan

\section{Institut für Hochenergiephysik, Wien, Austria}

W. Adam, F. Ambrogi, E. Asilar, T. Bergauer, J. Brandstetter, E. Brondolin, M. Dragicevic, J. Erö, A. Escalante Del Valle, M. Flechl, M. Friedl, R. Frühwirth ${ }^{1}$, V.M. Ghete, J. Grossmann, J. Hrubec, M. Jeitler ${ }^{1}$, A. König, N. Krammer, I. Krätschmer, D. Liko, T. Madlener, I. Mikulec, E. Pree, N. Rad, H. Rohringer, J. Schieck ${ }^{1}$, R. Schöfbeck, M. Spanring, D. Spitzbart, A. Taurok, W. Waltenberger, J. Wittmann, C.-E. Wulz ${ }^{1}$, M. Zarucki

\section{Institute for Nuclear Problems, Minsk, Belarus}

V. Chekhovsky, V. Mossolov, J. Suarez Gonzalez

\section{Universiteit Antwerpen, Antwerpen, Belgium}

E.A. De Wolf, D. Di Croce, X. Janssen, J. Lauwers, M. Pieters, M. Van De Klundert, H. Van Haevermaet, P. Van Mechelen, N. Van Remortel

\section{Vrije Universiteit Brussel, Brussel, Belgium}

S. Abu Zeid, F. Blekman, J. D'Hondt, I. De Bruyn, J. De Clercq, K. Deroover, G. Flouris, D. Lontkovskyi, S. Lowette, I. Marchesini, S. Moortgat, L. Moreels, Q. Python, K. Skovpen, S. Tavernier, W. Van Doninck, P. Van Mulders, I. Van Parijs

\section{Université Libre de Bruxelles, Bruxelles, Belgium}

D. Beghin, B. Bilin, H. Brun, B. Clerbaux, G. De Lentdecker, H. Delannoy, B. Dorney, G. Fasanella, L. Favart, R. Goldouzian, A. Grebenyuk, A.K. Kalsi, T. Lenzi, J. Luetic, T. Seva, E. Starling, C. Vander Velde, P. Vanlaer, D. Vannerom, R. Yonamine

\section{Ghent University, Ghent, Belgium}

T. Cornelis, D. Dobur, A. Fagot, M. Gul, I. Khvastunov², D. Poyraz, C. Roskas, D. Trocino, M. Tytgat, W. Verbeke, B. Vermassen, M. Vit, N. Zaganidis

\section{Université Catholique de Louvain, Louvain-la-Neuve, Belgium}

H. Bakhshiansohi, O. Bondu, S. Brochet, G. Bruno, C. Caputo, A. Caudron, P. David, S. De Visscher, C. Delaere, M. Delcourt, B. Francois, A. Giammanco, G. Krintiras, V. Lemaitre, A. Magitteri, A. Mertens, M. Musich, K. Piotrzkowski, L. Quertenmont, A. Saggio, M. Vidal Marono, S. Wertz, J. Zobec

\section{Centro Brasileiro de Pesquisas Fisicas, Rio de Janeiro, Brazil}

W.L. Aldá Júnior, F.L. Alves, G.A. Alves, L. Brito, G. Correia Silva, C. Hensel, A. Moraes, M.E. Pol, P. Rebello Teles

Universidade do Estado do Rio de Janeiro, Rio de Janeiro, Brazil

E. Belchior Batista Das Chagas, W. Carvalho, J. Chinellato ${ }^{3}$, E. Coelho, E.M. Da Costa, G.G. Da Silveira ${ }^{4}$, D. De Jesus Damiao, S. Fonseca De Souza, H. Malbouisson, M. Medina Jaime $^{5}$, M. Melo De Almeida, C. Mora Herrera, L. Mundim, H. Nogima, L.J. Sanchez 
Rosas, A. Santoro, A. Sznajder, M. Thiel, E.J. Tonelli Manganote ${ }^{3}$, F. Torres Da Silva De Araujo, A. Vilela Pereira

Universidade Estadual Paulista ${ }^{a}$, Universidade Federal do ABC $^{b}$, São Paulo, Brazil

S. Ahuja ${ }^{a}$, C.A. Bernardes ${ }^{a}$, L. Calligaris ${ }^{a}$, T.R. Fernandez Perez Tomei ${ }^{a}$, E.M. Gregores $^{b}$, P.G. Mercadante ${ }^{b}$, S.F. Novaes ${ }^{a}$, Sandra S. Padula ${ }^{a}$, D. Romero $\operatorname{Abad}^{b}$, J.C. Ruiz Vargas ${ }^{a}$

Institute for Nuclear Research and Nuclear Energy, Bulgarian Academy of Sciences, Sofia, Bulgaria

A. Aleksandrov, R. Hadjiiska, P. Iaydjiev, A. Marinov, M. Misheva, M. Rodozov, M. Shopova, G. Sultanov

University of Sofia, Sofia, Bulgaria

A. Dimitrov, L. Litov, B. Pavlov, P. Petkov

Beihang University, Beijing, China

W. Fang ${ }^{6}$, X. Gao ${ }^{6}$, L. Yuan

Institute of High Energy Physics, Beijing, China

M. Ahmad, J.G. Bian, G.M. Chen, H.S. Chen, M. Chen, Y. Chen, C.H. Jiang, D. Leggat,

H. Liao, Z. Liu, F. Romeo, S.M. Shaheen, A. Spiezia, J. Tao, C. Wang, Z. Wang, E. Yazgan,

H. Zhang, J. Zhao

State Key Laboratory of Nuclear Physics and Technology, Peking University, Beijing, China

Y. Ban, G. Chen, J. Li, Q. Li, S. Liu, Y. Mao, S.J. Qian, D. Wang, Z. Xu

Tsinghua University, Beijing, China

Y. Wang

Universidad de Los Andes, Bogota, Colombia

C. Avila, A. Cabrera, C.A. Carrillo Montoya, L.F. Chaparro Sierra, C. Florez, C.F. González Hernández, M.A. Segura Delgado

University of Split, Faculty of Electrical Engineering, Mechanical Engineering and Naval Architecture, Split, Croatia

B. Courbon, N. Godinovic, D. Lelas, I. Puljak, P.M. Ribeiro Cipriano, T. Sculac

University of Split, Faculty of Science, Split, Croatia

Z. Antunovic, M. Kovac

Institute Rudjer Boskovic, Zagreb, Croatia

V. Brigljevic, D. Ferencek, K. Kadija, B. Mesic, A. Starodumov ${ }^{7}$, T. Susa

University of Cyprus, Nicosia, Cyprus

M.W. Ather, A. Attikis, G. Mavromanolakis, J. Mousa, C. Nicolaou, F. Ptochos, P.A. Razis, H. Rykaczewski

Charles University, Prague, Czech Republic

M. Finger ${ }^{8}$, M. Finger Jr. ${ }^{8}$ 
Universidad San Francisco de Quito, Quito, Ecuador

E. Carrera Jarrin

Academy of Scientific Research and Technology of the Arab Republic of Egypt, Egyptian Network of High Energy Physics, Cairo, Egypt

H. Abdalla ${ }^{9}$, Y. Assran ${ }^{10,11}$, S. Elgammal ${ }^{11}$

National Institute of Chemical Physics and Biophysics, Tallinn, Estonia

S. Bhowmik, R.K. Dewanjee, M. Kadastik, L. Perrini, M. Raidal, C. Veelken

Department of Physics, University of Helsinki, Helsinki, Finland

P. Eerola, H. Kirschenmann, J. Pekkanen, M. Voutilainen

Helsinki Institute of Physics, Helsinki, Finland

J. Havukainen, J.K. Heikkilä, T. Järvinen, V. Karimäki, R. Kinnunen, T. Lampén,

K. Lassila-Perini, S. Laurila, S. Lehti, T. Lindén, P. Luukka, T. Mäenpää, H. Siikonen,

E. Tuominen, J. Tuominiemi

Lappeenranta University of Technology, Lappeenranta, Finland

T. Tuuva

IRFU, CEA, Université Paris-Saclay, Gif-sur-Yvette, France

M. Besancon, F. Couderc, M. Dejardin, D. Denegri, J.L. Faure, F. Ferri, S. Ganjour, S. Ghosh, A. Givernaud, P. Gras, G. Hamel de Monchenault, P. Jarry, C. Leloup, E. Locci, M. Machet, J. Malcles, G. Negro, J. Rander, A. Rosowsky, M.Ö. Sahin, M. Titov

Laboratoire Leprince-Ringuet, Ecole polytechnique, CNRS/IN2P3, Université Paris-Saclay, Palaiseau, France

A. Abdulsalam ${ }^{12}$, C. Amendola, I. Antropov, S. Baffioni, F. Beaudette, P. Busson,

L. Cadamuro, C. Charlot, R. Granier de Cassagnac, M. Jo, I. Kucher, S. Lisniak,

A. Lobanov, J. Martin Blanco, M. Nguyen, C. Ochando, G. Ortona, P. Paganini, P. Pigard,

R. Salerno, J.B. Sauvan, Y. Sirois, A.G. Stahl Leiton, Y. Yilmaz, A. Zabi, A. Zghiche

Université de Strasbourg, CNRS, IPHC UMR 7178, F-67000 Strasbourg, France

J.-L. Agram ${ }^{13}$, J. Andrea, D. Bloch, J.-M. Brom, E.C. Chabert, C. Collard, E. Conte ${ }^{13}$, X. Coubez, F. Drouhin ${ }^{13}$, J.-C. Fontaine ${ }^{13}$, D. Gelé, U. Goerlach, M. Jansová, P. Juillot, A.-C. Le Bihan, N. Tonon, P. Van Hove

Centre de Calcul de l'Institut National de Physique Nucleaire et de Physique des Particules, CNRS/IN2P3, Villeurbanne, France

S. Gadrat

Université de Lyon, Université Claude Bernard Lyon 1, CNRS-IN2P3, Institut de Physique Nucléaire de Lyon, Villeurbanne, France

S. Beauceron, C. Bernet, G. Boudoul, N. Chanon, R. Chierici, D. Contardo, P. Depasse, H. El Mamouni, J. Fay, L. Finco, S. Gascon, M. Gouzevitch, G. Grenier, B. Ille, F. Lagarde, I.B. Laktineh, H. Lattaud, M. Lethuillier, L. Mirabito, A.L. Pequegnot, S. Perries, A. Popov ${ }^{14}$, V. Sordini, M. Vander Donckt, S. Viret, S. Zhang 
Georgian Technical University, Tbilisi, Georgia

T. Toriashvili ${ }^{15}$

Tbilisi State University, Tbilisi, Georgia

Z. Tsamalaidze ${ }^{8}$

RWTH Aachen University, I. Physikalisches Institut, Aachen, Germany

C. Autermann, L. Feld, M.K. Kiesel, K. Klein, M. Lipinski, M. Preuten, M.P. Rauch, C. Schomakers, J. Schulz, M. Teroerde, B. Wittmer, V. Zhukov ${ }^{14}$

RWTH Aachen University, III. Physikalisches Institut A, Aachen, Germany

A. Albert, D. Duchardt, M. Endres, M. Erdmann, S. Erdweg, T. Esch, R. Fischer, A. Güth,

T. Hebbeker, C. Heidemann, K. Hoepfner, S. Knutzen, M. Merschmeyer, A. Meyer,

P. Millet, S. Mukherjee, T. Pook, M. Radziej, H. Reithler, M. Rieger, F. Scheuch,

D. Teyssier, S. Thüer

RWTH Aachen University, III. Physikalisches Institut B, Aachen, Germany

G. Flügge, B. Kargoll, T. Kress, A. Künsken, T. Müller, A. Nehrkorn, A. Nowack, C. Pistone, O. Pooth, A. Stahl ${ }^{16}$

\section{Deutsches Elektronen-Synchrotron, Hamburg, Germany}

M. Aldaya Martin, T. Arndt, C. Asawatangtrakuldee, K. Beernaert, O. Behnke, U. Behrens, A. Bermúdez Martínez, A.A. Bin Anuar, K. Borras ${ }^{17}$, V. Botta, A. Campbell, P. Connor, C. Contreras-Campana, F. Costanza, V. Danilov, A. De Wit, C. Diez Pardos, D. Domínguez Damiani, G. Eckerlin, D. Eckstein, T. Eichhorn, A. Elwood, E. Eren, E. Gallo ${ }^{18}$, J. Garay Garcia, A. Geiser, J.M. Grados Luyando, A. Grohsjean, P. Gunnellini, M. Guthoff, A. Harb, J. Hauk, H. Jung, M. Kasemann, J. Keaveney, C. Kleinwort, J. Knolle, I. Korol, D. Krücker, W. Lange, A. Lelek, T. Lenz, K. Lipka, W. Lohmann ${ }^{19}$, R. Mankel, I.-A. Melzer-Pellmann, A.B. Meyer, M. Meyer, M. Missiroli, G. Mittag, J. Mnich, A. Mussgiller, D. Pitzl, A. Raspereza, M. Savitskyi, P. Saxena, R. Shevchenko, N. Stefaniuk, H. Tholen, G.P. Van Onsem, R. Walsh, Y. Wen, K. Wichmann, C. Wissing, O. Zenaiev

\section{University of Hamburg, Hamburg, Germany}

R. Aggleton, S. Bein, V. Blobel, M. Centis Vignali, T. Dreyer, E. Garutti, D. Gonzalez, J. Haller, A. Hinzmann, M. Hoffmann, A. Karavdina, G. Kasieczka, R. Klanner, R. Kogler, N. Kovalchuk, S. Kurz, V. Kutzner, J. Lange, D. Marconi, J. Multhaup, M. Niedziela, D. Nowatschin, T. Peiffer, A. Perieanu, A. Reimers, C. Scharf, P. Schleper, A. Schmidt, S. Schumann, J. Schwandt, J. Sonneveld, H. Stadie, G. Steinbrück, F.M. Stober, M. Stöver, D. Troendle, E. Usai, A. Vanhoefer, B. Vormwald

\section{Institut für Experimentelle Teilchenphysik, Karlsruhe, Germany}

M. Akbiyik, C. Barth, M. Baselga, S. Baur, E. Butz, R. Caspart, T. Chwalek, F. Colombo, W. De Boer, A. Dierlamm, N. Faltermann, B. Freund, R. Friese, M. Giffels, M.A. Harrendorf, F. Hartmann ${ }^{16}$, S.M. Heindl, U. Husemann, F. Kassel ${ }^{16}$, S. Kudella, H. Mildner, M.U. Mozer, Th. Müller, M. Plagge, G. Quast, K. Rabbertz, M. Schröder, I. Shvetsov, 
G. Sieber, H.J. Simonis, R. Ulrich, S. Wayand, M. Weber, T. Weiler, S. Williamson, C. Wöhrmann, R. Wolf

Institute of Nuclear and Particle Physics (INPP), NCSR Demokritos, Aghia Paraskevi, Greece

G. Anagnostou, G. Daskalakis, T. Geralis, A. Kyriakis, D. Loukas, I. Topsis-Giotis

National and Kapodistrian University of Athens, Athens, Greece

G. Karathanasis, S. Kesisoglou, A. Panagiotou, N. Saoulidou, E. Tziaferi

National Technical University of Athens, Athens, Greece

K. Kousouris, I. Papakrivopoulos

University of Ioánnina, Ioánnina, Greece

I. Evangelou, C. Foudas, P. Gianneios, P. Katsoulis, P. Kokkas, S. Mallios, N. Manthos,

I. Papadopoulos, E. Paradas, J. Strologas, F.A. Triantis, D. Tsitsonis

MTA-ELTE Lendület CMS Particle and Nuclear Physics Group, Eötvös Loránd University, Budapest, Hungary

M. Csanad, N. Filipovic, G. Pasztor, O. Surányi, G.I. Veres

Wigner Research Centre for Physics, Budapest, Hungary

G. Bencze, C. Hajdu, D. Horvath ${ }^{20}$, Á. Hunyadi, F. Sikler, V. Veszpremi, G. Vesztergombi ${ }^{\dagger}$, T.Á. Vámi

Institute of Nuclear Research ATOMKI, Debrecen, Hungary

N. Beni, S. Czellar, J. Karancsi21, A. Makovec, J. Molnar, Z. Szillasi

Institute of Physics, University of Debrecen, Debrecen, Hungary

M. Bartók ${ }^{22}$, P. Raics, Z.L. Trocsanyi, B. Ujvari

Indian Institute of Science (IISc), Bangalore, India

S. Choudhury, J.R. Komaragiri

National Institute of Science Education and Research, Bhubaneswar, India

S. Bahinipatii ${ }^{23}$, P. Mal, K. Mandal, A. Nayak ${ }^{24}$, D.K. Sahoo ${ }^{23}$, S.K. Swain

Panjab University, Chandigarh, India

S. Bansal, S.B. Beri, V. Bhatnagar, S. Chauhan, R. Chawla, N. Dhingra, R. Gupta, A. Kaur, M. Kaur, S. Kaur, R. Kumar, P. Kumari, M. Lohan, A. Mehta, S. Sharma, J.B. Singh, G. Walia

University of Delhi, Delhi, India

Ashok Kumar, Aashaq Shah, A. Bhardwaj, B.C. Choudhary, R.B. Garg, S. Keshri, A. Kumar, S. Malhotra, M. Naimuddin, K. Ranjan, R. Sharma

Saha Institute of Nuclear Physics, HBNI, Kolkata, India

R. Bhardwaj ${ }^{25}$, R. Bhattacharya, S. Bhattacharya, U. Bhawandeep ${ }^{25}$, D. Bhowmik, S. Dey, S. Dutt ${ }^{25}$, S. Dutta, S. Ghosh, N. Majumdar, K. Mondal, S. Mukhopadhyay, S. Nandan, A. Purohit, P.K. Rout, A. Roy, S. Roy Chowdhury, S. Sarkar, M. Sharan, B. Singh, S. Thakur ${ }^{25}$ 
Indian Institute of Technology Madras, Madras, India

P.K. Behera

Bhabha Atomic Research Centre, Mumbai, India

R. Chudasama, D. Dutta, V. Jha, V. Kumar, A.K. Mohanty ${ }^{16}$, P.K. Netrakanti, L.M. Pant, P. Shukla, A. Topkar

Tata Institute of Fundamental Research-A, Mumbai, India

T. Aziz, S. Dugad, B. Mahakud, S. Mitra, G.B. Mohanty, N. Sur, B. Sutar

Tata Institute of Fundamental Research-B, Mumbai, India

S. Banerjee, S. Bhattacharya, S. Chatterjee, P. Das, M. Guchait, Sa. Jain, S. Kumar, M. Maity ${ }^{26}$, G. Majumder, K. Mazumdar, N. Sahoo, T. Sarkar ${ }^{26}$, N. Wickramage ${ }^{27}$

Indian Institute of Science Education and Research (IISER), Pune, India

S. Chauhan, S. Dube, V. Hegde, A. Kapoor, K. Kothekar, S. Pandey, A. Rane, S. Sharma

Institute for Research in Fundamental Sciences (IPM), Tehran, Iran

S. Chenarani ${ }^{28}$, E. Eskandari Tadavani, S.M. Etesami ${ }^{28}$, M. Khakzad, M. Mohammadi Najafabadi, M. Naseri, S. Paktinat Mehdiabadi ${ }^{29}$, F. Rezaei Hosseinabadi, B. Safarzadeh ${ }^{30}$, M. Zeinali

University College Dublin, Dublin, Ireland

M. Felcini, M. Grunewald

INFN Sezione di Bari ${ }^{a}$, Università di Bari ${ }^{b}$, Politecnico di Bari ${ }^{c}$, Bari, Italy M. Abbrescia ${ }^{a, b}$, C. Calabria ${ }^{a, b}$, A. Colaleo $^{a}$, D. Creanza ${ }^{a, c}$, L. Cristella $^{a, b}$, N. De Filippis ${ }^{a, c}$, M. De Palma ${ }^{a, b}$, A. Di Florio ${ }^{a, b}$, F. Errico ${ }^{a, b}$, L. Fiore ${ }^{a}$, A. Gelmi ${ }^{a, b}$, G. Iaselli ${ }^{a, c}$,

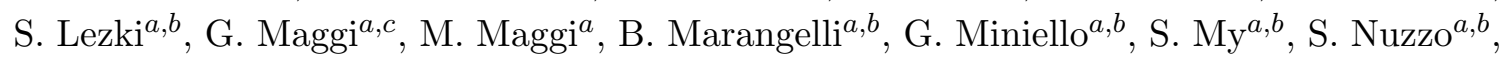
A. Pompili ${ }^{a, b}$, G. Pugliese ${ }^{a, c}$, R. Radogna ${ }^{a}$, A. Ranieri ${ }^{a}$, G. Selvaggi ${ }^{a, b}$, A. Sharma ${ }^{a}$, L. Silvestris ${ }^{a}, 16$, R. Venditti ${ }^{a}$, P. Verwilligen ${ }^{a}$, G. Zito ${ }^{a}$

INFN Sezione di Bologna ${ }^{a}$, Università di Bologna ${ }^{b}$, Bologna, Italy

G. Abbiendi ${ }^{a}$, C. Battilana ${ }^{a, b}$, D. Bonacorsi ${ }^{a, b}$, L. Borgonovi $^{a, b}$, S. Braibant-Giacomellia $^{a, b}$, L. Brigliadori ${ }^{a, b}$, R. Campanini ${ }^{a, b}$, P. Capiluppi ${ }^{a, b}$, A. Castro ${ }^{a, b}$, F.R. Cavallo ${ }^{a}$, S.S. Chhibra ${ }^{a, b}$, G. Codispoti ${ }^{a, b}$, M. Cuffiani ${ }^{a, b}$, G.M. Dallavalle ${ }^{a}$, F. Fabbri ${ }^{a}$, A. Fanfani ${ }^{a, b}$, D. Fasanella ${ }^{a, b}$, P. Giacomelli ${ }^{a}$, C. Grandi ${ }^{a}$, L. Guiducci ${ }^{a, b}$, S. Marcellini ${ }^{a}$, G. Masetti ${ }^{a}$,

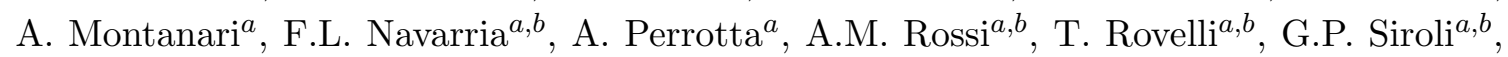
N. $\operatorname{Tosi}^{a}$

INFN Sezione di Catania ${ }^{a}$, Università di Catania ${ }^{b}$, Catania, Italy

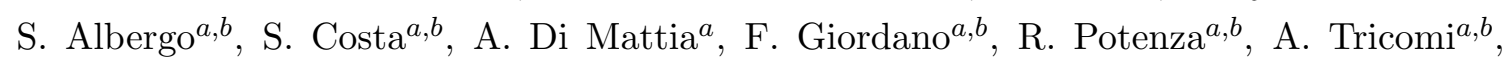
C. Tuve $\mathrm{e}^{a, b}$

INFN Sezione di Firenze ${ }^{a}$, Università di Firenze ${ }^{b}$, Firenze, Italy

G. Barbagli ${ }^{a}$, K. Chatterjee ${ }^{a, b}$, V. Ciulli ${ }^{a, b}$, C. Civinini ${ }^{a}$, R. D'Alessandro ${ }^{a, b}$, E. Focardi $^{a, b}$, G. Latino, P. Lenzi ${ }^{a, b}$, M. Meschini ${ }^{a}$, S. Paoletti $^{a}$, L. Russo $^{a}, 31$, G. Sguazzoni $^{a}$, D. Strom ${ }^{a}$, L. Viliani ${ }^{a}$ 
INFN Laboratori Nazionali di Frascati, Frascati, Italy

A. Alfonsi, L. Benussi, S. Bianco, F. Fabbri, D. Piccolo, F. Primavera ${ }^{16}$

INFN Sezione di Genova ${ }^{a}$, Università di Genova ${ }^{b}$, Genova, Italy

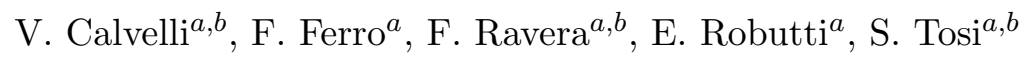

INFN Sezione di Milano-Bicocca ${ }^{a}$, Università di Milano-Bicocca ${ }^{b}$, Milano, $^{2}$ Italy

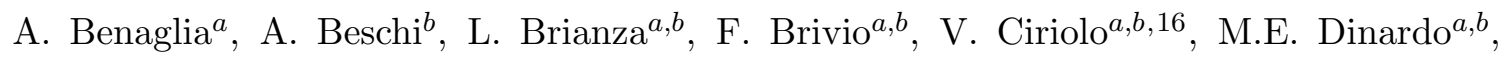
S. Fiorendi ${ }^{a, b}$, S. Gennai ${ }^{a}$, A. Ghezzi ${ }^{a, b}$, P. Govoni ${ }^{a, b}$, M. Malberti ${ }^{a, b}$, S. Malvezzi ${ }^{a}$, R.A. Manzoni ${ }^{a, b}$, D. Menasce ${ }^{a}$, L. Moroni ${ }^{a}$, M. Paganoni ${ }^{a, b}$, K. Pauwels ${ }^{a, b}$, D. Pedrini ${ }^{a}$, S. Pigazzini ${ }^{a, b, 32}$, S. Ragazzi ${ }^{a, b}$, T. Tabarelli de Fatis ${ }^{a, b}$

INFN Sezione di Napoli ${ }^{a}$, Università di Napoli 'Federico II' ${ }^{b}$, Napoli, Italy, Università della Basilicata ${ }^{c}$, Potenza, Italy, Università G. Marconi ${ }^{d}$, Roma, Italy

S. Buontempo ${ }^{a}$, N. Cavallo ${ }^{a, c}$, S. Di Guida ${ }^{a, d, 16}$, F. Fabozzi $^{a, c}$, F. Fienga ${ }^{a, b}$, G. Galati ${ }^{a, b}$,

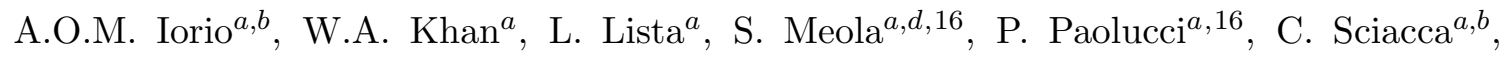
F. Thyssen ${ }^{a}$, E. Voevodina ${ }^{a, b}$

INFN Sezione di Padova ${ }^{a}$, Università di Padova ${ }^{b}$, Padova, Italy, Università di Trento $^{c}$, Trento, Italy

P. Azzi ${ }^{a}$, N. Bacchetta ${ }^{a}$, L. Benato ${ }^{a, b}$, D. Bisello ${ }^{a, b}$, A. Boletti ${ }^{a, b}$, R. Carlin ${ }^{a, b}$, A. Carvalho Antunes De Oliveira ${ }^{a, b}$, P. Checchia ${ }^{a}$, M. Dall'Osso ${ }^{a, b}$, P. De Castro Manzano ${ }^{a}$, T. Dorigo ${ }^{a}$, U. Dosselli ${ }^{a}$, F. Gasparini ${ }^{a, b}$, U. Gasparini $^{a}, b$, A. Gozzelino $^{a}$, S. Lacaprara ${ }^{a}$, P. Lujan, M. Margoni ${ }^{a, b}$, A.T. Meneguzzo ${ }^{a, b}$, N. Pozzobon ${ }^{a, b}$, P. Ronchese ${ }^{a, b}$, R. Rossin ${ }^{a, b}$, F. Simonetto ${ }^{a, b}$, A. Tiko, E. Torassa ${ }^{a}$, P. Zotto ${ }^{a, b}$, G. Zumerle $^{a, b}$

INFN Sezione di Pavia ${ }^{a}$, Università di Pavia ${ }^{b}$, Pavia, Italy

A. Braghieri ${ }^{a}$, A. Magnani ${ }^{a}$, P. Montagna ${ }^{a, b}$, S.P. Ratti ${ }^{a, b}$, V. $\operatorname{Re}^{a}$, M. Ressegotti ${ }^{a, b}$, C. Riccardi ${ }^{a, b}$, P. Salvini ${ }^{a}$, I. Vai ${ }^{a, b}$, P. Vitulo ${ }^{a, b}$

INFN Sezione di Perugia ${ }^{a}$, Università di Perugia ${ }^{b}$, Perugia, Italy

L. Alunni Solestizi ${ }^{a, b}$, M. Biasini ${ }^{a, b}$, G.M. Bilei ${ }^{a}$, C. Cecchi $^{a, b}$, D. Ciangottini ${ }^{a, b}$, L. Fanò $^{a, b}$, P. Lariccia ${ }^{a, b}$, R. Leonardi ${ }^{a, b}$, E. Manoni ${ }^{a}$, G. Mantovani ${ }^{a, b}$, V. Mariani $^{a}, b$, M. Menichelli ${ }^{a}$, A. $\operatorname{Rossi}^{a, b}$, A. Santocchia ${ }^{a, b}$, D. Spiga ${ }^{a}$

INFN Sezione di Pisa ${ }^{a}$, Università di $\mathrm{Pisa}^{b}$, Scuola Normale Superiore di Pisa ${ }^{c}$, Pisa, Italy

K. Androsov ${ }^{a}$, P. Azzurri ${ }^{a}$, G. Bagliesi ${ }^{a}$, L. Bianchini ${ }^{a}$, T. Boccali ${ }^{a}$, L. Borrello, R. Castaldi ${ }^{a}$, M.A. Ciocci ${ }^{a, b}$, R. Dell'Orso ${ }^{a}$, G. Fedi ${ }^{a}$, L. Giannini ${ }^{a, c}$, A. Giassi ${ }^{a}$, M.T. Grippo ${ }^{a}$, F. Ligabue ${ }^{a, c}$, T. Lomtadze ${ }^{a}$, E. Manca ${ }^{a, c}$, G. Mandorli ${ }^{a, c}$, A. Messineo ${ }^{a, b}$,

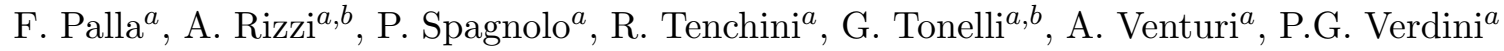

INFN Sezione di Roma ${ }^{a}$, Sapienza Università di Roma ${ }^{b}$, Rome, Italy

L. Barone ${ }^{a, b}$, F. Cavallari $^{a}$, M. Cipriani ${ }^{a, b}$, N. Daci ${ }^{a}$, D. Del Re Re $^{a, b}$, E. Di Marco ${ }^{a, b}$, M. Diemoz ${ }^{a}$, S. Gelli ${ }^{a, b}$, E. Longo ${ }^{a, b}$, B. Marzocchi ${ }^{a, b}$, P. Meridiani ${ }^{a}$, G. Organtini ${ }^{a, b}$,

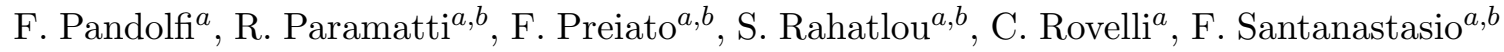


INFN Sezione di Torino ${ }^{a}$, Università di Torino ${ }^{b}$, Torino, Italy, Università del Piemonte Orientale ${ }^{c}$, Novara, Italy

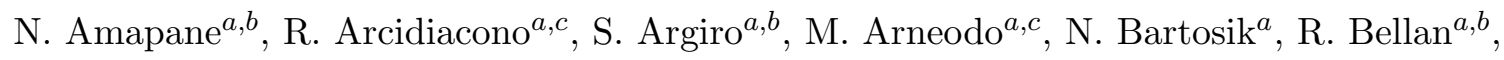
C. Biino ${ }^{a}$, N. Cartiglia ${ }^{a}$, R. Castello ${ }^{a, b}$, F. Cenna ${ }^{a, b}$, M. Costa ${ }^{a, b}$, R. Covarelli ${ }^{a, b}$, A. Degano ${ }^{a, b}$, N. $\operatorname{Demaria}^{a}$, B. Kiani ${ }^{a, b}$, C. Mariotti ${ }^{a}$, S. Maselli ${ }^{a}$, E. Migliore ${ }^{a, b}$, V. Monaco ${ }^{a, b}$, E. Monteil ${ }^{a, b}$, M. Monteno $^{a}$, M.M. Obertino ${ }^{a, b}$, L. Pacher $^{a, b}$, N. Pastrone $^{a}$, M. Pelliccioni ${ }^{a}$, G.L. Pinna Angioni ${ }^{a, b}$, A. Romero ${ }^{a, b}$, M. Ruspa ${ }^{a, c}$, R. Sacchi ${ }^{a, b}$, K. Shchelina ${ }^{a, b}$, V. Sola ${ }^{a}$, A. Solano ${ }^{a, b}$, A. Staiano ${ }^{a}$

INFN Sezione di Trieste ${ }^{a}$, Università di Trieste ${ }^{b}$, Trieste, Italy

S. Belforte ${ }^{a}$, M. Casarsa ${ }^{a}$, F. Cossutti ${ }^{a}$, G. Della Ricca ${ }^{a, b}$, A. Zanetti ${ }^{a}$

Kyungpook National University

D.H. Kim, G.N. Kim, M.S. Kim, J. Lee, S. Lee, S.W. Lee, C.S. Moon, Y.D. Oh, S. Sekmen, D.C. Son, Y.C. Yang

Chonnam National University, Institute for Universe and Elementary Particles, Kwangju, Korea

H. Kim, D.H. Moon, G. Oh

Hanyang University, Seoul, Korea

J.A. Brochero Cifuentes, J. Goh, T.J. Kim

Korea University, Seoul, Korea

S. Cho, S. Choi, Y. Go, D. Gyun, S. Ha, B. Hong, Y. Jo, Y. Kim, K. Lee, K.S. Lee, S. Lee, J. Lim, S.K. Park, Y. Roh

Seoul National University, Seoul, Korea

J. Almond, J. Kim, J.S. Kim, H. Lee, K. Lee, K. Nam, M. Oh, S.B. Oh, B.C. RadburnSmith, S.h. Seo, U.K. Yang, H.D. Yoo, G.B. Yu

University of Seoul, Seoul, Korea

H. Kim, J.H. Kim, J.S.H. Lee, I.C. Park

Sungkyunkwan University, Suwon, Korea

Y. Choi, C. Hwang, J. Lee, I. Yu

Vilnius University, Vilnius, Lithuania

V. Dudenas, A. Juodagalvis, J. Vaitkus

National Centre for Particle Physics, Universiti Malaya, Kuala Lumpur, Malaysia

I. Ahmed, Z.A. Ibrahim, M.A.B. Md Ali ${ }^{33}$, F. Mohamad Idris ${ }^{34}$, W.A.T. Wan Abdullah, M.N. Yusli, Z. Zolkapli

Centro de Investigacion y de Estudios Avanzados del IPN, Mexico City, Mexico Reyes-Almanza, R, Ramirez-Sanchez, G., Duran-Osuna, M. C., H. Castilla-Valdez, E. De La Cruz-Burelo, I. Heredia-De La Cruz ${ }^{35}$, Rabadan-Trejo, R. I., R. Lopez-Fernandez, J. Mejia Guisao, A. Sanchez-Hernandez 
Universidad Iberoamericana, Mexico City, Mexico

S. Carrillo Moreno, C. Oropeza Barrera, F. Vazquez Valencia

Benemerita Universidad Autonoma de Puebla, Puebla, Mexico

J. Eysermans, I. Pedraza, H.A. Salazar Ibarguen, C. Uribe Estrada

Universidad Autónoma de San Luis Potosí, San Luis Potosí, Mexico

A. Morelos Pineda

University of Auckland, Auckland, New Zealand

D. Krofcheck

University of Canterbury, Christchurch, New Zealand

S. Bheesette, P.H. Butler

National Centre for Physics, Quaid-I-Azam University, Islamabad, Pakistan

A. Ahmad, M. Ahmad, Q. Hassan, H.R. Hoorani, A. Saddique, M.A. Shah, M. Shoaib, M. Waqas

National Centre for Nuclear Research, Swierk, Poland

H. Bialkowska, M. Bluj, B. Boimska, T. Frueboes, M. Górski, M. Kazana, K. Nawrocki, M. Szleper, P. Traczyk, P. Zalewski

Institute of Experimental Physics, Faculty of Physics, University of Warsaw, Warsaw, Poland

K. Bunkowski, A. Byszuk ${ }^{36}$, K. Doroba, A. Kalinowski, M. Konecki, J. Krolikowski, M. Misiura, M. Olszewski, A. Pyskir, M. Walczak

Laboratório de Instrumentação e Física Experimental de Partículas, Lisboa, Portugal

P. Bargassa, C. Beirão Da Cruz E Silva, A. Di Francesco, P. Faccioli, B. Galinhas, M. Gallinaro, J. Hollar, N. Leonardo, L. Lloret Iglesias, M.V. Nemallapudi, J. Seixas, G. Strong, O. Toldaiev, D. Vadruccio, J. Varela

Joint Institute for Nuclear Research, Dubna, Russia

S. Afanasiev, P. Bunin, M. Gavrilenko, I. Golutvin, I. Gorbunov, A. Kamenev, V. Karjavin, A. Lanev, A. Malakhov, V. Matveev ${ }^{37,38}$, P. Moisenz, V. Palichik, V. Perelygin, S. Shmatov, S. Shulha, N. Skatchkov, V. Smirnov, N. Voytishin, A. Zarubin

Petersburg Nuclear Physics Institute, Gatchina (St. Petersburg), Russia

Y. Ivanov, V. Kim ${ }^{39}$, E. Kuznetsova ${ }^{40}$, P. Levchenko, V. Murzin, V. Oreshkin, I. Smirnov, D. Sosnov, V. Sulimov, L. Uvarov, S. Vavilov, A. Vorobyev

Institute for Nuclear Research, Moscow, Russia

Yu. Andreev, A. Dermenev, S. Gninenko, N. Golubev, A. Karneyeu, M. Kirsanov, N. Krasnikov, A. Pashenkov, D. Tlisov, A. Toropin

Institute for Theoretical and Experimental Physics, Moscow, Russia

V. Epshteyn, V. Gavrilov, N. Lychkovskaya, V. Popov, I. Pozdnyakov, G. Safronov, A. Spiridonov, A. Stepennov, V. Stolin, M. Toms, E. Vlasov, A. Zhokin 
Moscow Institute of Physics and Technology, Moscow, Russia

T. Aushev, A. Bylinkin ${ }^{38}$

National Research Nuclear University 'Moscow Engineering Physics Institute' (MEPhI), Moscow, Russia

M. Chadeeva ${ }^{41}$, P. Parygin, D. Philippov, S. Polikarpov, E. Popova, V. Rusinov

P.N. Lebedev Physical Institute, Moscow, Russia

V. Andreev, M. Azarkin ${ }^{38}$, I. Dremin ${ }^{38}$, M. Kirakosyan ${ }^{38}$, S.V. Rusakov, A. Terkulov

Skobeltsyn Institute of Nuclear Physics, Lomonosov Moscow State University, Moscow, Russia

A. Baskakov, A. Belyaev, E. Boos, M. Dubinin ${ }^{42}$, L. Dudko, A. Ershov, A. Gribushin, V. Klyukhin, O. Kodolova, I. Lokhtin, I. Miagkov, S. Obraztsov, S. Petrushanko, V. Savrin, A. Snigirev

Novosibirsk State University (NSU), Novosibirsk, Russia V. Blinov ${ }^{43}$, D. Shtol ${ }^{43}$, Y. Skovpen ${ }^{43}$

State Research Center of Russian Federation, Institute for High Energy Physics of NRC \&quot;Kurchatov Institute\&quot;, Protvino, Russia

I. Azhgirey, I. Bayshev, S. Bitioukov, D. Elumakhov, A. Godizov, V. Kachanov, A. Kalinin,

D. Konstantinov, P. Mandrik, V. Petrov, R. Ryutin, A. Sobol, S. Troshin, N. Tyurin,

A. Uzunian, A. Volkov

National Research Tomsk Polytechnic University, Tomsk, Russia

A. Babaev

University of Belgrade, Faculty of Physics and Vinca Institute of Nuclear Sciences, Belgrade, Serbia

P. Adzic ${ }^{44}$, P. Cirkovic, D. Devetak, M. Dordevic, J. Milosevic

Centro de Investigaciones Energéticas Medioambientales y Tecnológicas (CIEMAT), Madrid, Spain

J. Alcaraz Maestre, I. Bachiller, M. Barrio Luna, M. Cerrada, N. Colino, B. De La Cruz, A. Delgado Peris, C. Fernandez Bedoya, J.P. Fernández Ramos, J. Flix, M.C. Fouz, O. Gonzalez Lopez, S. Goy Lopez, J.M. Hernandez, M.I. Josa, D. Moran, A. Pérez-Calero Yzquierdo, J. Puerta Pelayo, I. Redondo, L. Romero, M.S. Soares, A. Triossi, A. Álvarez Fernández

Universidad Autónoma de Madrid, Madrid, Spain

C. Albajar, J.F. de Trocóniz

Universidad de Oviedo, Oviedo, Spain

J. Cuevas, C. Erice, J. Fernandez Menendez, S. Folgueras, I. Gonzalez Caballero, J.R. González Fernández, E. Palencia Cortezon, S. Sanchez Cruz, P. Vischia, J.M. Vizan Garcia 
Instituto de Física de Cantabria (IFCA), CSIC-Universidad de Cantabria, Santander, Spain

I.J. Cabrillo, A. Calderon, B. Chazin Quero, J. Duarte Campderros, M. Fernandez, P.J. Fernández Manteca, J. Garcia-Ferrero, A. García Alonso, G. Gomez, A. Lopez Virto, J. Marco, C. Martinez Rivero, P. Martinez Ruiz del Arbol, F. Matorras, J. Piedra Gomez, C. Prieels, T. Rodrigo, A. Ruiz-Jimeno, L. Scodellaro, N. Trevisani, I. Vila, R. Vilar Cortabitarte

\section{CERN, European Organization for Nuclear Research, Geneva, Switzerland}

D. Abbaneo, B. Akgun, E. Auffray, P. Baillon, A.H. Ball, D. Barney, J. Bendavid, M. Bianco, A. Bocci, C. Botta, T. Camporesi, M. Cepeda, G. Cerminara, E. Chapon, Y. Chen, D. d'Enterria, A. Dabrowski, V. Daponte, A. David, M. De Gruttola, A. De Roeck, N. Deelen, M. Dobson, T. du Pree, M. Dünser, N. Dupont, A. Elliott-Peisert, P. Everaerts, F. Fallavollita ${ }^{45}$, G. Franzoni, J. Fulcher, W. Funk, D. Gigi, A. Gilbert, K. Gill, F. Glege, D. Gulhan, J. Hegeman, V. Innocente, A. Jafari, P. Janot, O. Karacheban ${ }^{19}$, J. Kieseler, V. Knünz, A. Kornmayer, M. Krammer ${ }^{1}$, C. Lange, P. Lecoq, C. Lourenço, M.T. Lucchini, L. Malgeri, M. Mannelli, A. Martelli, F. Meijers, J.A. Merlin, S. Mersi, E. Meschi, P. Milenovic ${ }^{46}$, F. Moortgat, M. Mulders, H. Neugebauer, J. Ngadiuba, S. Orfanelli, L. Orsini, F. Pantaleo ${ }^{16}$, L. Pape, E. Perez, M. Peruzzi, A. Petrilli, G. Petrucciani, A. Pfeiffer, M. Pierini, F.M. Pitters, D. Rabady, A. Racz, T. Reis, G. Rolandi ${ }^{47}$, M. Rovere, H. Sakulin, C. Schäfer, C. Schwick, M. Seidel, M. Selvaggi, A. Sharma, P. Silva, P. Sphicas ${ }^{48}$, A. Stakia, J. Steggemann, M. Stoye, M. Tosi, D. Treille, A. Tsirou, V. Veckalns ${ }^{49}$, M. Verweij, W.D. Zeuner

\section{Paul Scherrer Institut, Villigen, Switzerland}

W. Bertl ${ }^{\dagger}$, L. Caminada ${ }^{50}$, K. Deiters, W. Erdmann, R. Horisberger, Q. Ingram, H.C. Kaestli, D. Kotlinski, U. Langenegger, T. Rohe, S.A. Wiederkehr

ETH Zurich - Institute for Particle Physics and Astrophysics (IPA), Zurich, Switzerland

M. Backhaus, L. Bäni, P. Berger, B. Casal, N. Chernyavskaya, G. Dissertori, M. Dittmar, M. Donegà, C. Dorfer, C. Grab, C. Heidegger, D. Hits, J. Hoss, T. Klijnsma, W. Lustermann, M. Marionneau, M.T. Meinhard, D. Meister, F. Micheli, P. Musella, F. NessiTedaldi, J. Pata, F. Pauss, G. Perrin, L. Perrozzi, M. Quittnat, M. Reichmann, D. Ruini, D.A. Sanz Becerra, M. Schönenberger, L. Shchutska, V.R. Tavolaro, K. Theofilatos, M.L. Vesterbacka Olsson, R. Wallny, D.H. Zhu

\section{Universität Zürich, Zurich, Switzerland}

T.K. Aarrestad, C. Amsler ${ }^{51}$, D. Brzhechko, M.F. Canelli, A. De Cosa, R. Del Burgo, S. Donato, C. Galloni, T. Hreus, B. Kilminster, I. Neutelings, D. Pinna, G. Rauco, P. Robmann, D. Salerno, K. Schweiger, C. Seitz, Y. Takahashi, A. Zucchetta

\section{National Central University, Chung-Li, Taiwan}

V. Candelise, Y.H. Chang, K.y. Cheng, T.H. Doan, Sh. Jain, R. Khurana, C.M. Kuo, W. Lin, A. Pozdnyakov, S.S. Yu 
National Taiwan University (NTU), Taipei, Taiwan

Arun Kumar, P. Chang, Y. Chao, K.F. Chen, P.H. Chen, F. Fiori, W.-S. Hou, Y. Hsiung, Y.F. Liu, R.-S. Lu, E. Paganis, A. Psallidas, A. Steen, J.f. Tsai

Chulalongkorn University, Faculty of Science, Department of Physics, Bangkok, Thailand

B. Asavapibhop, K. Kovitanggoon, G. Singh, N. Srimanobhas

Çukurova University, Physics Department, Science and Art Faculty, Adana, Turkey

A. Bat, F. Boran, S. Cerci ${ }^{52}$, S. Damarseckin, Z.S. Demiroglu, C. Dozen, I. Dumanoglu,

S. Girgis, G. Gokbulut, Y. Guler, I. Hos ${ }^{53}$, E.E. Kangal ${ }^{54}$, O. Kara, A. Kayis Topaksu,

U. Kiminsu, M. Oglakci, G. Onengut, K. Ozdemir ${ }^{55}$, D. Sunar Cerci ${ }^{52}$, U.G. Tok,

H. Topakli ${ }^{56}$, S. Turkcapar, I.S. Zorbakir, C. Zorbilmez

Middle East Technical University, Physics Department, Ankara, Turkey

G. Karapinar ${ }^{57}$, K. Ocalan ${ }^{58}$, M. Yalvac, M. Zeyrek

Bogazici University, Istanbul, Turkey

I.O. Atakisi, E. Gülmez, M. Kaya ${ }^{59}$, O. Kaya ${ }^{60}$, S. Tekten, E.A. Yetkin ${ }^{61}$

Istanbul Technical University, Istanbul, Turkey

M.N. Agaras, S. Atay, A. Cakir, K. Cankocak, Y. Komurcu

Institute for Scintillation Materials of National Academy of Science of Ukraine, Kharkov, Ukraine

B. Grynyov

National Scientific Center, Kharkov Institute of Physics and Technology, Kharkov, Ukraine

L. Levchuk

University of Bristol, Bristol, United Kingdom

F. Ball, L. Beck, J.J. Brooke, D. Burns, E. Clement, D. Cussans, O. Davignon, H. Flacher,

J. Goldstein, G.P. Heath, H.F. Heath, L. Kreczko, D.M. Newbold ${ }^{62}$, S. Paramesvaran,

T. Sakuma, S. Seif El Nasr-storey, D. Smith, V.J. Smith

Rutherford Appleton Laboratory, Didcot, United Kingdom

K.W. Bell, A. Belyaev ${ }^{63}$, C. Brew, R.M. Brown, D. Cieri, D.J.A. Cockerill, J.A. Coughlan, K. Harder, S. Harper, J. Linacre, E. Olaiya, D. Petyt, C.H. Shepherd-Themistocleous, A. Thea, I.R. Tomalin, T. Williams, W.J. Womersley

Imperial College, London, United Kingdom

G. Auzinger, R. Bainbridge, P. Bloch, J. Borg, S. Breeze, O. Buchmuller, A. Bundock, S. Casasso, D. Colling, L. Corpe, P. Dauncey, G. Davies, M. Della Negra, R. Di Maria, Y. Haddad, G. Hall, G. Iles, T. James, M. Komm, R. Lane, C. Laner, L. Lyons, A.-M. Magnan, S. Malik, L. Mastrolorenzo, T. Matsushita, J. Nash ${ }^{64}$, A. Nikitenko ${ }^{7}$, V. Palladino, M. Pesaresi, A. Richards, A. Rose, E. Scott, C. Seez, A. Shtipliyski, 
T. Strebler, S. Summers, A. Tapper, K. Uchida, M. Vazquez Acosta ${ }^{65}$, T. Virdee ${ }^{16}$, N. Wardle, D. Winterbottom, J. Wright, S.C. Zenz

Brunel University, Uxbridge, United Kingdom

J.E. Cole, P.R. Hobson, A. Khan, P. Kyberd, A. Morton, I.D. Reid, L. Teodorescu, S. Zahid

Baylor University, Waco, U.S.A.

A. Borzou, K. Call, J. Dittmann, K. Hatakeyama, H. Liu, N. Pastika, C. Smith

Catholic University of America, Washington DC, U.S.A.

R. Bartek, A. Dominguez

The University of Alabama, Tuscaloosa, U.S.A.

A. Buccilli, S.I. Cooper, C. Henderson, P. Rumerio, C. West

Boston University, Boston, U.S.A.

D. Arcaro, A. Avetisyan, T. Bose, D. Gastler, D. Rankin, C. Richardson, J. Rohlf, L. Sulak,

D. Zou

\section{Brown University, Providence, U.S.A.}

G. Benelli, D. Cutts, M. Hadley, J. Hakala, U. Heintz, J.M. Hogan ${ }^{66}$, K.H.M. Kwok,

E. Laird, G. Landsberg, J. Lee, Z. Mao, M. Narain, J. Pazzini, S. Piperov, S. Sagir,

R. Syarif, D. Yu

University of California, Davis, Davis, U.S.A.

R. Band, C. Brainerd, R. Breedon, D. Burns, M. Calderon De La Barca Sanchez, M. Chertok, J. Conway, R. Conway, P.T. Cox, R. Erbacher, C. Flores, G. Funk, W. Ko, R. Lander, C. Mclean, M. Mulhearn, D. Pellett, J. Pilot, S. Shalhout, M. Shi, J. Smith, D. Stolp, D. Taylor, K. Tos, M. Tripathi, Z. Wang, F. Zhang

University of California, Los Angeles, U.S.A.

M. Bachtis, C. Bravo, R. Cousins, A. Dasgupta, A. Florent, J. Hauser, M. Ignatenko, N. Mccoll, S. Regnard, D. Saltzberg, C. Schnaible, V. Valuev

University of California, Riverside, Riverside, U.S.A.

E. Bouvier, K. Burt, R. Clare, J. Ellison, J.W. Gary, S.M.A. Ghiasi Shirazi, G. Hanson,

G. Karapostoli, E. Kennedy, F. Lacroix, O.R. Long, M. Olmedo Negrete, M.I. Paneva, W. Si, L. Wang, H. Wei, S. Wimpenny, B. R. Yates

University of California, San Diego, La Jolla, U.S.A.

J.G. Branson, S. Cittolin, M. Derdzinski, R. Gerosa, D. Gilbert, B. Hashemi, A. Holzner, D. Klein, G. Kole, V. Krutelyov, J. Letts, M. Masciovecchio, D. Olivito, S. Padhi, M. Pieri, M. Sani, V. Sharma, S. Simon, M. Tadel, A. Vartak, S. Wasserbaech ${ }^{67}$, J. Wood, F. Würthwein, A. Yagil, G. Zevi Della Porta

University of California, Santa Barbara - Department of Physics, Santa Barbara, U.S.A.

N. Amin, R. Bhandari, J. Bradmiller-Feld, C. Campagnari, M. Citron, A. Dishaw, V. Dutta, M. Franco Sevilla, L. Gouskos, R. Heller, J. Incandela, A. Ovcharova, H. Qu, J. Richman, D. Stuart, I. Suarez, J. Yoo 
California Institute of Technology, Pasadena, U.S.A.

D. Anderson, A. Bornheim, J. Bunn, J.M. Lawhorn, H.B. Newman, T. Q. Nguyen, C. Pena, M. Spiropulu, J.R. Vlimant, R. Wilkinson, S. Xie, Z. Zhang, R.Y. Zhu

\section{Carnegie Mellon University, Pittsburgh, U.S.A.}

M.B. Andrews, T. Ferguson, T. Mudholkar, M. Paulini, J. Russ, M. Sun, H. Vogel, I. Vorobiev, M. Weinberg

University of Colorado Boulder, Boulder, U.S.A.

J.P. Cumalat, W.T. Ford, F. Jensen, A. Johnson, M. Krohn, S. Leontsinis, E. MacDonald, T. Mulholland, K. Stenson, K.A. Ulmer, S.R. Wagner

Cornell University, Ithaca, U.S.A.

J. Alexander, J. Chaves, Y. Cheng, J. Chu, A. Datta, K. Mcdermott, N. Mirman, J.R. Patterson, D. Quach, A. Rinkevicius, A. Ryd, L. Skinnari, L. Soffi, S.M. Tan, Z. Tao, J. Thom, J. Tucker, P. Wittich, M. Zientek

\section{Fermi National Accelerator Laboratory, Batavia, U.S.A.}

S. Abdullin, M. Albrow, M. Alyari, G. Apollinari, A. Apresyan, A. Apyan, S. Banerjee, L.A.T. Bauerdick, A. Beretvas, J. Berryhill, P.C. Bhat, G. Bolla ${ }^{\dagger}$, K. Burkett, J.N. Butler, A. Canepa, G.B. Cerati, H.W.K. Cheung, F. Chlebana, M. Cremonesi, J. Duarte, V.D. Elvira, J. Freeman, Z. Gecse, E. Gottschalk, L. Gray, D. Green, S. Grünendahl, O. Gutsche, J. Hanlon, R.M. Harris, S. Hasegawa, J. Hirschauer, Z. Hu, B. Jayatilaka, S. Jindariani, M. Johnson, U. Joshi, B. Klima, M.J. Kortelainen, B. Kreis, S. Lammel, D. Lincoln, R. Lipton, M. Liu, T. Liu, R. Lopes De Sá, J. Lykken, K. Maeshima, N. Magini, J.M. Marraffino, D. Mason, P. McBride, P. Merkel, S. Mrenna, S. Nahn, V. O'Dell, K. Pedro, O. Prokofyev, G. Rakness, L. Ristori, A. Savoy-Navarro ${ }^{68}$, B. Schneider, E. Sexton-Kennedy, A. Soha, W.J. Spalding, L. Spiegel, S. Stoynev, J. Strait, N. Strobbe, L. Taylor, S. Tkaczyk, N.V. Tran, L. Uplegger, E.W. Vaandering, C. Vernieri, M. Verzocchi, R. Vidal, M. Wang, H.A. Weber, A. Whitbeck, W. Wu

University of Florida, Gainesville, U.S.A.

D. Acosta, P. Avery, P. Bortignon, D. Bourilkov, A. Brinkerhoff, A. Carnes, M. Carver, D. Curry, R.D. Field, I.K. Furic, S.V. Gleyzer, B.M. Joshi, J. Konigsberg, A. Korytov, K. Kotov, P. Ma, K. Matchev, H. Mei, G. Mitselmakher, K. Shi, D. Sperka, N. Terentyev, L. Thomas, J. Wang, S. Wang, J. Yelton

\section{Florida International University, Miami, U.S.A.}

Y.R. Joshi, S. Linn, P. Markowitz, J.L. Rodriguez

Florida State University, Tallahassee, U.S.A.

A. Ackert, T. Adams, A. Askew, S. Hagopian, V. Hagopian, K.F. Johnson, T. Kolberg, G. Martinez, T. Perry, H. Prosper, A. Saha, A. Santra, V. Sharma, R. Yohay

Florida Institute of Technology, Melbourne, U.S.A.

M.M. Baarmand, V. Bhopatkar, S. Colafranceschi, M. Hohlmann, D. Noonan, T. Roy, F. Yumiceva 
University of Illinois at Chicago (UIC), Chicago, U.S.A.

M.R. Adams, L. Apanasevich, D. Berry, R.R. Betts, R. Cavanaugh, X. Chen, S. Dittmer, O. Evdokimov, C.E. Gerber, D.A. Hangal, D.J. Hofman, K. Jung, J. Kamin, I.D. Sandoval Gonzalez, M.B. Tonjes, N. Varelas, H. Wang, Z. Wu, J. Zhang

The University of Iowa, Iowa City, U.S.A.

B. Bilki ${ }^{69}$, W. Clarida, K. Dilsiz ${ }^{70}$, S. Durgut, R.P. Gandrajula, M. Haytmyradov, V. Khristenko, J.-P. Merlo, H. Mermerkaya ${ }^{71}$, A. Mestvirishvili, A. Moeller, J. Nachtman, H. Ogul ${ }^{72}$, Y. Onel, F. Ozok ${ }^{73}$, A. Penzo, C. Snyder, E. Tiras, J. Wetzel, K. Yi

Johns Hopkins University, Baltimore, U.S.A.

B. Blumenfeld, A. Cocoros, N. Eminizer, D. Fehling, L. Feng, A.V. Gritsan, W.T. Hung, P. Maksimovic, J. Roskes, U. Sarica, M. Swartz, M. Xiao, C. You

The University of Kansas, Lawrence, U.S.A.

A. Al-bataineh, P. Baringer, A. Bean, S. Boren, J. Bowen, J. Castle, S. Khalil, A. Kropivnitskaya, D. Majumder, W. Mcbrayer, M. Murray, C. Rogan, C. Royon, S. Sanders, E. Schmitz, J.D. Tapia Takaki, Q. Wang

Kansas State University, Manhattan, U.S.A.

A. Ivanov, K. Kaadze, Y. Maravin, A. Modak, A. Mohammadi, L.K. Saini, N. Skhirtladze

Lawrence Livermore National Laboratory, Livermore, U.S.A.

F. Rebassoo, D. Wright

University of Maryland, College Park, U.S.A.

A. Baden, O. Baron, A. Belloni, S.C. Eno, Y. Feng, C. Ferraioli, N.J. Hadley, S. Jabeen, G.Y. Jeng, R.G. Kellogg, J. Kunkle, A.C. Mignerey, F. Ricci-Tam, Y.H. Shin, A. Skuja, S.C. Tonwar

\section{Massachusetts Institute of Technology, Cambridge, U.S.A.}

D. Abercrombie, B. Allen, V. Azzolini, R. Barbieri, A. Baty, G. Bauer, R. Bi, S. Brandt, W. Busza, I.A. Cali, M. D'Alfonso, Z. Demiragli, G. Gomez Ceballos, M. Goncharov, P. Harris, D. Hsu, M. Hu, Y. Iiyama, G.M. Innocenti, M. Klute, D. Kovalskyi, Y.-J. Lee, A. Levin, P.D. Luckey, B. Maier, A.C. Marini, C. Mcginn, C. Mironov, S. Narayanan, X. Niu, C. Paus, C. Roland, G. Roland, G.S.F. Stephans, K. Sumorok, K. Tatar, D. Velicanu, J. Wang, T.W. Wang, B. Wyslouch, S. Zhaozhong

University of Minnesota, Minneapolis, U.S.A.

A.C. Benvenuti, R.M. Chatterjee, A. Evans, P. Hansen, S. Kalafut, Y. Kubota, Z. Lesko, J. Mans, S. Nourbakhsh, N. Ruckstuhl, R. Rusack, J. Turkewitz, M.A. Wadud

University of Mississippi, Oxford, U.S.A.

J.G. Acosta, S. Oliveros

University of Nebraska-Lincoln, Lincoln, U.S.A.

E. Avdeeva, K. Bloom, D.R. Claes, C. Fangmeier, F. Golf, R. Gonzalez Suarez, R. Kamalieddin, I. Kravchenko, J. Monroy, J.E. Siado, G.R. Snow, B. Stieger 
State University of New York at Buffalo, Buffalo, U.S.A.

A. Godshalk, C. Harrington, I. Iashvili, D. Nguyen, A. Parker, S. Rappoccio, B. Roozbahani Northeastern University, Boston, U.S.A.

G. Alverson, E. Barberis, C. Freer, A. Hortiangtham, A. Massironi, D.M. Morse, T. Orimoto, R. Teixeira De Lima, T. Wamorkar, B. Wang, A. Wisecarver, D. Wood

Northwestern University, Evanston, U.S.A.

S. Bhattacharya, O. Charaf, K.A. Hahn, N. Mucia, N. Odell, M.H. Schmitt, K. Sung, M. Trovato, M. Velasco

University of Notre Dame, Notre Dame, U.S.A.

R. Bucci, N. Dev, M. Hildreth, K. Hurtado Anampa, C. Jessop, D.J. Karmgard, N. Kellams,

K. Lannon, W. Li, N. Loukas, N. Marinelli, F. Meng, C. Mueller, Y. Musienko ${ }^{37}$, M. Planer,

A. Reinsvold, R. Ruchti, P. Siddireddy, G. Smith, S. Taroni, M. Wayne, A. Wightman, M. Wolf, A. Woodard

The Ohio State University, Columbus, U.S.A.

J. Alimena, L. Antonelli, B. Bylsma, L.S. Durkin, S. Flowers, B. Francis, A. Hart, C. Hill, W. Ji, T.Y. Ling, W. Luo, B.L. Winer, H.W. Wulsin

Princeton University, Princeton, U.S.A.

S. Cooperstein, O. Driga, P. Elmer, J. Hardenbrook, P. Hebda, S. Higginbotham, A. Kalogeropoulos, D. Lange, J. Luo, D. Marlow, K. Mei, I. Ojalvo, J. Olsen, C. Palmer, P. Piroué, J. Salfeld-Nebgen, D. Stickland, C. Tully

University of Puerto Rico, Mayaguez, U.S.A.

S. Malik, S. Norberg

Purdue University, West Lafayette, U.S.A.

A. Barker, V.E. Barnes, S. Das, L. Gutay, M. Jones, A.W. Jung, A. Khatiwada, D.H. Miller, N. Neumeister, C.C. Peng, H. Qiu, J.F. Schulte, J. Sun, F. Wang, R. Xiao, W. Xie

Purdue University Northwest, Hammond, U.S.A.

T. Cheng, J. Dolen, N. Parashar

Rice University, Houston, U.S.A.

Z. Chen, K.M. Ecklund, S. Freed, F.J.M. Geurts, M. Guilbaud, M. Kilpatrick, W. Li, B. Michlin, B.P. Padley, J. Roberts, J. Rorie, W. Shi, Z. Tu, J. Zabel, A. Zhang

University of Rochester, Rochester, U.S.A.

A. Bodek, P. de Barbaro, R. Demina, Y.t. Duh, T. Ferbel, M. Galanti, A. Garcia-Bellido, J. Han, O. Hindrichs, A. Khukhunaishvili, K.H. Lo, P. Tan, M. Verzetti

The Rockefeller University, New York, U.S.A.

R. Ciesielski, K. Goulianos, C. Mesropian

Rutgers, The State University of New Jersey, Piscataway, U.S.A.

A. Agapitos, J.P. Chou, Y. Gershtein, T.A. Gómez Espinosa, E. Halkiadakis, M. Heindl,

E. Hughes, S. Kaplan, R. Kunnawalkam Elayavalli, S. Kyriacou, A. Lath, R. Montalvo, 
K. Nash, M. Osherson, H. Saka, S. Salur, S. Schnetzer, D. Sheffield, S. Somalwar, R. Stone, S. Thomas, P. Thomassen, M. Walker

\section{University of Tennessee, Knoxville, U.S.A.}

A.G. Delannoy, J. Heideman, G. Riley, K. Rose, S. Spanier, K. Thapa

\section{Texas A\&M University, College Station, U.S.A.}

O. Bouhali ${ }^{74}$, A. Castaneda Hernandez ${ }^{74}$, A. Celik, M. Dalchenko, M. De Mattia, A. Delgado, S. Dildick, R. Eusebi, J. Gilmore, T. Huang, T. Kamon ${ }^{75}$, R. Mueller, Y. Pakhotin, R. Patel, A. Perloff, L. Perniè, D. Rathjens, A. Safonov, A. Tatarinov

\section{Texas Tech University, Lubbock, U.S.A.}

N. Akchurin, J. Damgov, F. De Guio, P.R. Dudero, J. Faulkner, E. Gurpinar, S. Kunori, K. Lamichhane, S.W. Lee, T. Mengke, S. Muthumuni, T. Peltola, S. Undleeb, I. Volobouev, Z. Wang

\section{Vanderbilt University, Nashville, U.S.A.}

S. Greene, A. Gurrola, R. Janjam, W. Johns, C. Maguire, A. Melo, H. Ni, K. Padeken, J.D. Ruiz Alvarez, P. Sheldon, S. Tuo, J. Velkovska, Q. Xu

\section{University of Virginia, Charlottesville, U.S.A.}

M.W. Arenton, P. Barria, B. Cox, R. Hirosky, M. Joyce, A. Ledovskoy, H. Li, C. Neu, T. Sinthuprasith, Y. Wang, E. Wolfe, F. Xia

\section{Wayne State University, Detroit, U.S.A.}

R. Harr, P.E. Karchin, N. Poudyal, J. Sturdy, P. Thapa, S. Zaleski

\section{University of Wisconsin - Madison, Madison, WI, U.S.A.}

M. Brodski, J. Buchanan, C. Caillol, D. Carlsmith, S. Dasu, L. Dodd, S. Duric, B. Gomber, M. Grothe, M. Herndon, A. Hervé, U. Hussain, P. Klabbers, A. Lanaro, A. Levine, K. Long, R. Loveless, V. Rekovic, T. Ruggles, A. Savin, N. Smith, W.H. Smith, N. Woods

\section{†: Deceased}

1: Also at Vienna University of Technology, Vienna, Austria

2: Also at IRFU, CEA, Université Paris-Saclay, Gif-sur-Yvette, France

3: Also at Universidade Estadual de Campinas, Campinas, Brazil

4: Also at Federal University of Rio Grande do Sul, Porto Alegre, Brazil

5: Also at Universidade Federal de Pelotas, Pelotas, Brazil

6: Also at Université Libre de Bruxelles, Bruxelles, Belgium

7: Also at Institute for Theoretical and Experimental Physics, Moscow, Russia

8: Also at Joint Institute for Nuclear Research, Dubna, Russia

9: Also at Cairo University, Cairo, Egypt

10: Also at Suez University, Suez, Egypt

11: Now at British University in Egypt, Cairo, Egypt

12: Also at Department of Physics, King Abdulaziz University, Jeddah, Saudi Arabia

13: Also at Université de Haute Alsace, Mulhouse, France

14: Also at Skobeltsyn Institute of Nuclear Physics, Lomonosov Moscow State University, Moscow, Russia 
15: Also at Tbilisi State University, Tbilisi, Georgia

16: Also at CERN, European Organization for Nuclear Research, Geneva, Switzerland

17: Also at RWTH Aachen University, III. Physikalisches Institut A, Aachen, Germany

18: Also at University of Hamburg, Hamburg, Germany

19: Also at Brandenburg University of Technology, Cottbus, Germany

20: Also at Institute of Nuclear Research ATOMKI, Debrecen, Hungary

21: Also at Institute of Physics, University of Debrecen, Debrecen, Hungary

22: Also at MTA-ELTE Lendület CMS Particle and Nuclear Physics Group, Eötvös Loránd University, Budapest, Hungary

23: Also at Indian Institute of Technology Bhubaneswar, Bhubaneswar, India

24: Also at Institute of Physics, Bhubaneswar, India

25: Also at Shoolini University, Solan, India

26: Also at University of Visva-Bharati, Santiniketan, India

27: Also at University of Ruhuna, Matara, Sri Lanka

28: Also at Isfahan University of Technology, Isfahan, Iran

29: Also at Yazd University, Yazd, Iran

30: Also at Plasma Physics Research Center, Science and Research Branch, Islamic Azad University, Tehran, Iran

31: Also at Università degli Studi di Siena, Siena, Italy

32: Also at INFN Sezione di Milano-Bicocca; Università di Milano-Bicocca, Milano, Italy

33: Also at International Islamic University of Malaysia, Kuala Lumpur, Malaysia

34: Also at Malaysian Nuclear Agency, MOSTI, Kajang, Malaysia

35: Also at Consejo Nacional de Ciencia y Tecnología, Mexico city, Mexico

36: Also at Warsaw University of Technology, Institute of Electronic Systems, Warsaw, Poland

37: Also at Institute for Nuclear Research, Moscow, Russia

38: Now at National Research Nuclear University 'Moscow Engineering Physics Institute' (MEPhI), Moscow, Russia

39: Also at St. Petersburg State Polytechnical University, St. Petersburg, Russia

40: Also at University of Florida, Gainesville, U.S.A.

41: Also at P.N. Lebedev Physical Institute, Moscow, Russia

42: Also at California Institute of Technology, Pasadena, U.S.A.

43: Also at Budker Institute of Nuclear Physics, Novosibirsk, Russia

44: Also at Faculty of Physics, University of Belgrade, Belgrade, Serbia

45: Also at INFN Sezione di Pavia; Università di Pavia, Pavia, Italy

46: Also at University of Belgrade, Faculty of Physics and Vinca Institute of Nuclear Sciences, Belgrade, Serbia

47: Also at Scuola Normale e Sezione dell'INFN, Pisa, Italy

48: Also at National and Kapodistrian University of Athens, Athens, Greece

49: Also at Riga Technical University, Riga, Latvia

50: Also at Universität Zürich, Zurich, Switzerland

51: Also at Stefan Meyer Institute for Subatomic Physics (SMI), Vienna, Austria

52: Also at Adiyaman University, Adiyaman, Turkey

53: Also at Istanbul Aydin University, Istanbul, Turkey

54: Also at Mersin University, Mersin, Turkey

55: Also at Piri Reis University, Istanbul, Turkey

56: Also at Gaziosmanpasa University, Tokat, Turkey

57: Also at Izmir Institute of Technology, Izmir, Turkey

58: Also at Necmettin Erbakan University, Konya, Turkey 
59: Also at Marmara University, Istanbul, Turkey

60: Also at Kafkas University, Kars, Turkey

61: Also at Istanbul Bilgi University, Istanbul, Turkey

62: Also at Rutherford Appleton Laboratory, Didcot, United Kingdom

63: Also at School of Physics and Astronomy, University of Southampton, Southampton, United Kingdom

64: Also at Monash University, Faculty of Science, Clayton, Australia

65: Also at Instituto de Astrofísica de Canarias, La Laguna, Spain

66: Also at Bethel University, ST. PAUL, U.S.A.

67: Also at Utah Valley University, Orem, U.S.A.

68: Also at Purdue University, West Lafayette, U.S.A.

69: Also at Beykent University, Istanbul, Turkey

70: Also at Bingol University, Bingol, Turkey

71: Also at Erzincan University, Erzincan, Turkey

72: Also at Sinop University, Sinop, Turkey

73: Also at Mimar Sinan University, Istanbul, Istanbul, Turkey

74: Also at Texas A\&M University at Qatar, Doha, Qatar

75: Also at Kyungpook National University, Daegu, Korea 